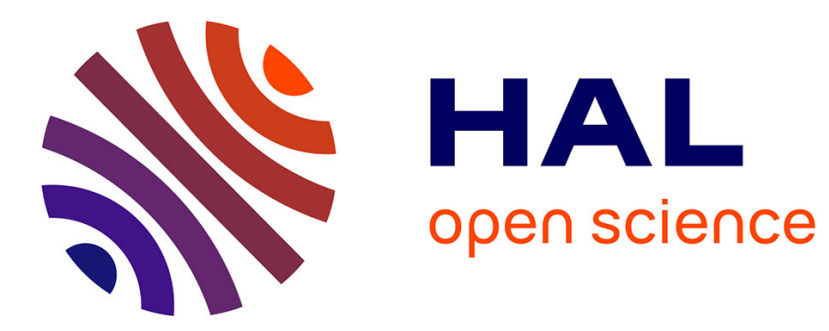

\title{
Secondary peak in the Nusselt number distribution of impinging jet flows: A phenomenological analysis
}

\author{
P Aillaud, Florent Duchaine, L. Gicquel, S. Didorally
}

\section{To cite this version:}

P Aillaud, Florent Duchaine, L. Gicquel, S. Didorally. Secondary peak in the Nusselt number distribution of impinging jet flows: A phenomenological analysis. Physics of Fluids, 2016, 28. hal-02571553

\section{HAL Id: hal-02571553 \\ https://hal.science/hal-02571553}

Submitted on 12 May 2020

HAL is a multi-disciplinary open access archive for the deposit and dissemination of scientific research documents, whether they are published or not. The documents may come from teaching and research institutions in France or abroad, or from public or private research centers.
L'archive ouverte pluridisciplinaire $\mathbf{H A L}$, est destinée au dépôt et à la diffusion de documents scientifiques de niveau recherche, publiés ou non, émanant des établissements d'enseignement et de recherche français ou étrangers, des laboratoires publics ou privés. 


\section{AIP $\left.\right|_{\text {Physics of }}$ Fluids}

Secondary peak in the Nusselt number distribution of impinging jet flows: A phenomenological analysis

P. Aillaud, F. Duchaine, L. Y. M. Gicquel, and S. Didorally

Citation: Physics of Fluids 28, 095110 (2016); doi: 10.1063/1.4963687

View online: http://dx.doi.org/10.1063/1.4963687

View Table of Contents: http://scitation.aip.org/content/aip/journal/pof2/28/9?ver=pdfcov

Published by the AIP Publishing

\section{Articles you may be interested in}

Large eddy simulation of the near-field vortex dynamics in starting square jet transitioning into steady state

Phys. Fluids 28, 085104 (2016); 10.1063/1.4961199

Round impinging jets with relatively large stand-off distance

Phys. Fluids 28, 075107 (2016); 10.1063/1.4955167

Detailed flowfield and surface properties for high Knudsen number planar jet impingement at an inclined flat plate

Phys. Fluids 28, 056103 (2016); 10.1063/1.4948365

Statistical analysis of the velocity and scalar fields in reacting turbulent wall-jets

Phys. Fluids 27, 025102 (2015); 10.1063/1.4906370

The inviscid impingement of a jet with arbitrary velocity profile

Phys. Fluids 12, 2046 (2000); 10.1063/1.870450

\section{Looking for a specific} instrument?

Easy access to the latest equipment.

Shop the Physics Today Buyer's Guide.

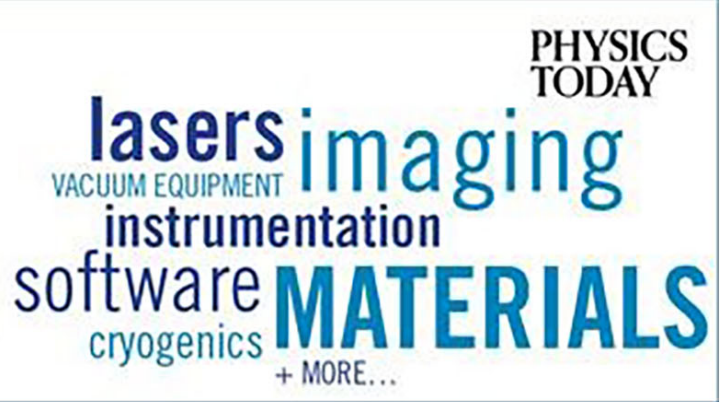




\title{
Secondary peak in the Nusselt number distribution of impinging jet flows: A phenomenological analysis
}

\author{
P. Aillaud, ${ }^{1, a)}$ F. Duchaine,${ }^{1}$ L. Y. M. Gicquel, ${ }^{1}$ and S. Didorally ${ }^{2}$ \\ ${ }^{1}$ Centre Européen de Recherche et de Formation Avancée en Calcul Scientifique (CERFACS), \\ CFD Team, 42 Avenue Gaspard Coriolis, 31057 Toulouse, France \\ ${ }^{2}$ SAFRAN Helicopter Engines, 64510 Bordes, France
}

(Received 19 April 2016; accepted 13 September 2016; published online 30 September 2016)

\begin{abstract}
This paper focuses on a wall-resolved Large Eddy Simulation (LES) of an isothermal round submerged air jet impinging on a heated flat plate, at a Reynolds number of 23000 (based on the nozzle diameter and the bulk velocity at the nozzle outlet) and for a nozzle to plate distance of two jet diameters. This specific configuration is known to lead to a non-monotonic variation of the temporal-mean Nusselt number as a function of the jet center distance, with the presence of two distinct peaks located on the jet axis and close to two nozzle diameters from the jet axis. The objectives are here twofold: first, validate the LES results against experimental data available in the literature and second to explore this validated numerical database by the use of high order statistics such as skewness and probability density functions of the temporal distribution of temperature and pressure to identify flow features at the origin of the second Nusselt peak. Skewness (Sk) of the pressure temporal distribution reveals the rebound of the primary vortices located near the location of the secondary peak and allows to identify the initiation of the unsteady separation linked to the local minimum in the mean heat transfer distribution. In the region of mean heat transfer enhancement, joint velocity-temperature analyses highlight that the most probable event is a cold fluid flux towards the plate produced by the passage of the vortical structures. In parallel, heat transfer distributions, analyzed using similar statistical tools, allow to connect the above mentioned events to the heat transfer on the plate. Thanks to such advanced analyses, the origin of the double peak is confirmed and connected to the flow dynamics. Published by AIP Publishing. [http://dx.doi.org/10.1063/1.4963687]
\end{abstract}

\section{INTRODUCTION}

In the aeronautical context, economical and environmental constraints have led engine manufacturers to work on the enhancement of the thermodynamic engine efficiency. With current technologies, this is achieved by increasing the Turbine Entry Temperature (TET) which makes the cooling of High Pressure (HP) turbine mandatory to control the lifetime of the blades. ${ }^{1}$ Due to the high heat transfer rate it can produce, impinging jet is one of the most common techniques used to cool the HP vanes. A common practice for engine manufacturers is to use empirical correlations for the preliminary design phase to determine several bulk parameters, e.g., Reynolds number and hole diameter, inherent to the design of such a cooling system. However, these correlations are less suitable when one wants to achieve an optimal design as they do not contain any physical information. To achieve this, a detailed knowledge of the underlying physical phenomena is therefore required involving a return to basic flow configurations as, for example, the impinging jet on a flat plate.

In this context, submerged impinging jet flows have attracted the interest of many researchers as can be seen from several literature reviews. ${ }^{2-4}$ Despite the apparent geometric simplicity, impinging jet flows are actually quite complex as they are composed of different zones, each containing

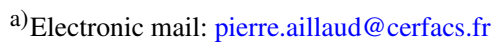


different dynamics with interlinked effects. The macroscopic description of impinging jets with low nozzle to plate distance involves three distinct regions. ${ }^{5}$ In the free jet region, the jet is not affected by the plate and the flow is mainly axial. The stagnation region is characterized by a deflection of the initially purely axial flow in the radial direction. This region generates a favorable pressure gradient that causes local flow acceleration. Finally in the wall jet region, the flow is mainly radial and the radial velocity initially increasing from $0 \mathrm{~m} / \mathrm{s}$ at the stagnation point reaches a maximum further downstream. Due to mass conservation, this region of strong flow acceleration also induces a reduction of the wall jet thickness. Then, the radial velocity starts to decrease and the wall jet gets thicker. Several flow visualizations of impinging jets have revealed the development of large scale primary structures within the shear layer of the free jet region due to the mean velocity gradient between the jet and the ambient air. ${ }^{6-8}$ As the primary vortical structures travel downstream in the free jet region, they are subject to multiple interactions and vortex pairings can occur. The coalescence of two or more vortex rings increases the size of the structure and decreases the passage frequency. Note also that the initial jet vortex passing frequency can vary considerably with the distance from the nozzle exit or the Reynolds number. However, it seems that sufficiently far from the nozzle lip (at a distance larger than $5 D$ downstream the nozzle lip, where $D$ is the jet diameter), the jet tends to forget the initial conditions at the nozzle outlet and reported large scale Strouhal numbers tend to a universal value ${ }^{9}$ of $S t_{D}=f D / U_{b}=0.3$, where $U_{b}$ is the bulk velocity at the jet outlet. This universal value is commonly referred as the "jet preferred mode." ${ }^{10}$ When the jet to plate distance decreases below 4, current consensus identifies two kind of vortical structures in impinging jet flows. First, the so-called primary vortices are induced by the roll-up of the free jet shear layer discussed above and issued by the growth of the Kelvin-Helmholtz instability near the nozzle exit. As for single vortex ring impinging on a wall, these primary vortices are stretched in the radial direction as they approach the wall. ${ }^{11-13}$ The local interaction of the vortex with the wall induces an adverse pressure gradient and a local unsteady separation of the boundary layer. ${ }^{14}$ This separation evolves then into a counter-rotating secondary vortex (with respect to the primary vortices).

Heat transfer characteristics of impinging jets have been extensively studied experimentally ${ }^{5,15-20}$ and numerically. ${ }^{13,21-24}$ For a single jet, there are three main parameters that govern heat transfer: the Reynolds number, ${ }^{17} R e=U_{b} D / v$, where $v$ is the kinematic viscosity, the nozzle to plate distance ${ }^{16,25} H$, and the mean velocity profile at the nozzle outlet ${ }^{26-28}$ which is different for different nozzle geometries for example. For "sufficiently high" Reynolds numbers and low nozzle to plate distance, i.e., $H / D<4$, one interesting feature is the non-monotonic variation of the radial distribution of the mean wall heat transfer with the presence of two distinct peaks. ${ }^{25}$ The first peak is related to the maximum mean heat transfer and is generally located at the stagnation point or near $r / D=0.5$ while the secondary peak occurs near $r / D=2$, where $r$ is the radial distance from the jet axis. Previous studies have shown that the first peak at the stagnation point and the high heat transfer rate in the stagnation region, i.e., for $r / D<0.5$, are caused by the jet flapping process associated with the vortex rings formed in the free jet shear layer. ${ }^{22,29}$ The origin of the secondary maximum was attributed for a long time to the turbulent-laminar transition of the wall jet. ${ }^{17,25}$ It was also shown that the location of the secondary peak is closely linked to the location of the maximum of the root mean square (RMS) velocity fluctuations. ${ }^{19,30}$ From these studies, a link was proposed between turbulence and the secondary peak without making the distinction between the possible coherent fluctuations induced by the passage of the large scale coherent structures and the stochastic fluctuations related to turbulence. More recently, Roux et al. ${ }^{20}$ performed unsteady measurements of wall temperature fluctuations using infrared thermography and evidenced the propagation of warm and cold fronts with propagation velocities very close to the vortices convection velocity. Hadžiabdić and Hanjalićc ${ }^{22}$ performed a Large Eddy Simulation (LES) of a single round, normally impinging jet issuing from a long pipe for a nozzle to plate distance $H=2 D$ at $R e=20000$. They attributed the secondary peak to the unsteady reattachment of the boundary layer, while the dip between the two peaks is believed to be caused by the unsteady separation of the boundary layer. Instantaneous analyses of low Reynolds number impinging jets ${ }^{31,32}$ have also shown a link between the location of the primary and secondary vortices and local heat transfer variations. Another interesting point highlighted by Rohlfs et al. ${ }^{32}$ for a laminar forced impinging jet using instantaneous visualizations is the ejection of the vortices that takes place near the location of the secondary peak. However, as mentioned by these authors, these 
instantaneous analyses certainly give important clues but do not provide a full explanation of what happens in a time-averaged view. Dairay et al. ${ }^{21}$ conducted the first Direct Numerical Simulation (DNS) of a confined round impinging jet in a relatively high Reynolds number case, $R e=10000$ and $H=2 D$. They were able to observe the primary vortices formed in the free jet shear layer as well as the secondary vortices generated near the wall. The secondary peak was related to strong and highly intermittent thermal events associated with negative wall shear stress events used to characterize the near wall backward flow that can be connected to the secondary vortices.

From this survey, it appears clearly today that a link exists between large scale coherent structures and heat transfer. However, it is still difficult to fully understand the physical mechanisms that are behind the mean heat transfer enhancement process. Hence, the main goal of this study is to provide additional information to characterize the physics using LES. The paper begins with the specification of the flow configuration and a description of the available experimental data in terms of heat transfer and flow dynamics. Then, the numerical methods are described, uncertainties related to LES are quantified, and the results are compared to the available experimental data. This leads to the conclusion that the results obtained from this simulation can be used confidently to analyze the physics. After a description of the general organization of the flow field, the LES database is used to construct high order statistics such as skewness and kurtosis, jointly with probability density functions (PDFs) of the temporal distribution of pressure, axial velocity, and temperature to highlight the trajectory of the primary and secondary vortices as well as their effect on the near wall behavior. Finally, the wall heat transfer is analyzed and compared to the flow dynamics highlighting the effective link with the previous events evidenced by the use of PDFs and high order moments.

\section{FLOW CONFIGURATION AND AVAILABLE EXPERIMENTAL DATA}

A schematic of the jet impingement configuration investigated here is presented in Fig. 1. It is an unconfined three dimensional turbulent subsonic isothermal round jet normally impinging on a hot flat plate. The nozzle to plate distance $H$ is 2 times the diameter of the jet $D$ and the Reynolds number based on the bulk velocity and $D$ is 23000 . The Mach number is $M=U_{b} / c=0.1$, where $c$ is the sound speed. This specific configuration is known to lead to a double peak in the plate Nusselt number distribution. ${ }^{13,16,22,24}$ Several experimental studies have dealt with this setup and heat transfer results are not always in agreement. For example, for similar flow configurations and similar injection nozzles, i.e., long straight round pipe, the first peak in the radial Nusselt distribution is either found at the stagnation point ${ }^{16,27,33}$ or near $r / D=0.5 .^{25}$ In the literature, it appears that the

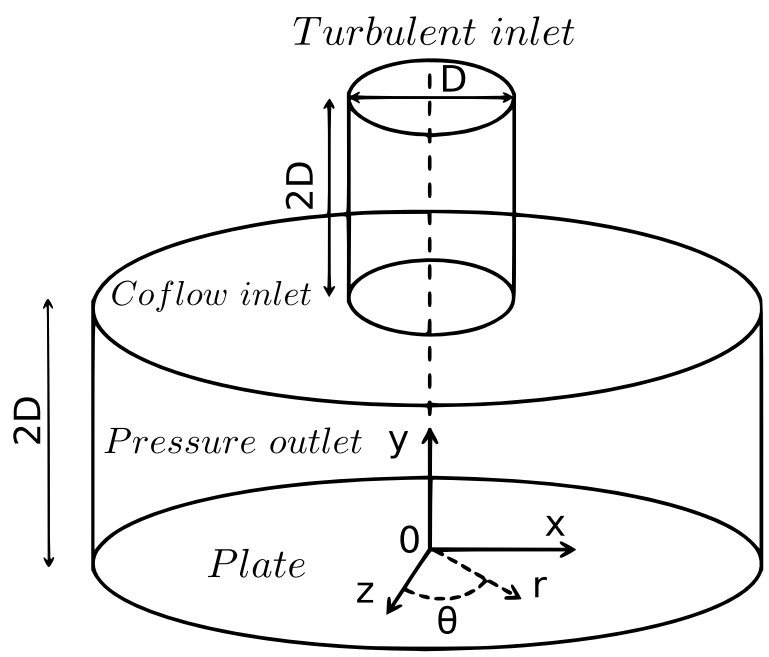

FIG. 1. Schematic view of the flow configuration with associated coordinate system: the origin is located on the plate at the nozzle geometrical center and the $y$ axis is normal to the plate pointing inward; $r$ is the radial distance from the origin and $\theta$ the azimuthal angle ( $\theta=0$ is placed along the $x$ axis). 
mean velocity profile at the nozzle outlet, mainly influenced by the nozzle geometry, is one of the main parameters affecting the radial Nusselt distribution for small nozzle to plate spacings as shown experimentally ${ }^{26,27}$ and numerically. ${ }^{21,32}$ Note also that except for recent studies, ${ }^{34}$ it is difficult to find, in the existing literature, comprehensive experimental databases reporting at the same time the flow dynamics and the heat transfer. This makes an accurate comparison between experimental and numerical results difficult as it leads to a combination of different experiments with different experimental facilities for the validation process of numerical simulations. For this reason, two different data sets are used here for the validation of the flow dynamics and heat transfer.

The experimental data from Tummers, Jacobse, and Voorbrood, ${ }^{35}$ obtained for an impinging jet on a flat plate at $R e=23000$ and a nozzle to plate distance $H=2 D$, are here used for the validation of the flow dynamics. The injection nozzle is a straight pipe and has a length of $76 \mathrm{D}$ leading to a fully developed velocity profile at the outlet. The velocity profiles are measured at several radial locations using the two component Laser Doppler Anemometry (LDA) technique. High resolution one component LDA technique is also used to perform velocity measurements very close to the wall. These near wall measurements along with a linear fit to the mean radial velocity allow to determine the radial wall shear stress at several radial locations. For the validation of the thermal results, the data of Fenot, Vullierme, and Dorignac ${ }^{33}$ obtained for a cold jet impinging on a quasi-isothermal hot plate at $R e=23000$ and $H / D=2$, are used as their boundary condition on the plate is similar to what is done in the LES.

\section{NUMERICAL METHODS}

This section is dedicated to the description of the numerical methods used for this study. The solver is first described along with the inherent approximations, issued by the numerical schemes, and the various turbulence closures or models. Then, the treatment of boundary conditions is discussed and numerical uncertainties related to the spatial discretization and the Sub-Grid Scale (SGS) modeling are specifically illustrated.

\section{A. Solver}

The parallel LES solver AVBP ${ }^{36}$ developed by CERFACS and IFP-EN is used to solve the 3D filtered compressible Navier-Stokes equations. A finite element two-step time-explicit Taylor Galerkin scheme (TTGC) ${ }^{37}$ is used for the discretization of the convective terms based on a cell-vertex formulation. The scheme provides a third-order accuracy in time and space while ensuring low dispersion and diffusion properties which is in agreement with the requirements of LES applications. ${ }^{37}$ A second order Galerkin scheme is used for diffusion terms. ${ }^{38}$ Such numerics are especially designed for LES on hybrid meshes and have been extensively validated in the context of turbulent flow applications. ${ }^{39-44}$ The explicit temporal integration is the major drawback of this strategy because the time step $\Delta t$ is limited, for stability reasons, by the acoustic Courant Friedrichs Lewy number defined as $C F L=(u+c) \Delta t / \Delta x$, where $\Delta x$ and $u$ are, respectively, the local mesh size and flow velocity $(C F L=0.7$ for this study and $\Delta t \approx 0.4 \mu \mathrm{s}$ for the finest mesh) leading to a relatively high computational cost. The unresolved Sub-Grid Scale (SGS) stress tensor is modeled using the Boussinesq assumption, ${ }^{45}$ and the SGS viscosity is computed with the Wall Adapting Local Eddy-viscosity (WALE) model that is well suited for wall-resolved LES $^{46}$ as it recovers the proper $y^{3}$ near-wall scaling of the eddy viscosity contrarily to the more conventional Smagorinsky model. ${ }^{47}$ The SGS heat flux vector is modeled using the classical gradient-diffusion hypothesis ${ }^{45}$ that relates the SGS heat flux to the filtered temperature gradient using a SGS thermal conductivity. This approach postulates a direct analogy between the momentum and heat transfer through the SGS turbulent Prandtl number $\left(P r_{S G S}=\mu_{S G S} C_{p} / \lambda_{S G S}\right)$, here fixed at $\operatorname{Pr}_{S G S}=0.5$. To assess the effect of the turbulent Prandtl number value, a simulation with $P r_{S G S}=0.9$ was also performed. Due to the LES wall-resolved approach chosen here, this has only a small effect on heat transfer results in the stagnation region. The maximum relative difference, 
$\left|N u\left(\operatorname{Pr}_{S G S}=0.5\right)-N u\left(\operatorname{Pr}_{S G S}=0.9\right)\right| / N u\left(\operatorname{Pr}_{S G S}=0.5\right)$, is $4 \%$ and locates at the stagnation point. For $r / D>0.25$, the relative difference between the two cases is less than $2 \%$.

\section{B. Boundary conditions}

A mean velocity profile is imposed at the inlet along with a uniform temperature, $T_{\infty}=300 \mathrm{~K}$, using the Navier-Stokes Characteristic Boundary Condition (NSCBC) formalism. ${ }^{48}$ The axial velocity is prescribed using a power law profile for turbulent pipe flows ${ }^{49}$ given in Eq. (1) following the recommendation of Cooper et al. ${ }^{50}$ while the other components are set to zero,

$$
\frac{U(r)}{U_{c}}=\left(1-\frac{2 r}{D}\right)^{1 / 7.23}
$$

where $U_{c}$ is the centerline velocity given by Eq. (2) and $r$ represents the distance to the jet axis,

$$
\frac{U_{b}}{U_{c}}=0.811+0.038(\log (R e)-4) \text {. }
$$

To mimic the turbulent flow developing in the nozzle, isotropic velocity fluctuations are injected at the inlet using a Passot-Pouquet spectrum and following a non-reflecting formalism to avoid numerical noise. ${ }^{51}$ The most energetic length scale that defines the peak in the spectrum is set to $D / 3$ and the amplitude of the injected velocity fluctuations is set to $0.05 U_{b}$. For unconfined impinging jets, a specific numerical treatment of the top free boundary is required. Setting the static pressure on this top boundary and treating it as an outlet is not suitable as massive inflow occurs in such configuration due to the entrainment of ambient air by the jet. Another possibility is to impose a weak constant and perpendicular co-flow directed towards the plate that does not impact the development of the impinging jet. Such a boundary condition is not completely irrelevant from a physical point of view, since present in the experiment and as long as its effect on the jet is minimal. Note that this flow characterization is usually not provided but can be indirectly observed in experiments as seen, for example, in Fig. 4(b) where the axial velocity goes to $0.03 U_{b}$ outside of the wall jet in the experiment of Tummers, Jacobse, and Voorbrood. ${ }^{35}$ Ideally, measured mean co-flow velocity profiles should be imposed but such information is not provided neither by Tummers, Jacobse, and Voorbrood ${ }^{35}$ nor by Fenot, Vullierme, and Dorignac ${ }^{33}$ which adds to the uncertainties of the various experimental databases. Several co-flow intensities have been tested here ranging from $2 \%$ to $10 \%$ of the bulk velocity, and the velocity magnitude was finally fixed at $5 \%$ of the jet bulk velocity as it was the highest velocity that does not influence the jet dynamics while preserving the control of the simulation. One can note that this intensity is small compared to the jet velocity that acts as a shield protecting the plate from any influence of the co-flow. These modeling issues comply with existing studies that have also shown that such moderate co-flow velocity does not impact the jet behavior. ${ }^{22}$ At the outlet, the static pressure is enforced using the NSCBC formalism accounting for the transverse terms. ${ }^{52}$ The nozzle wall is adiabatic with a no-slip condition imposed. The plate is treated as an isothermal no-slip wall with a wall temperature, $T_{w}=330 \mathrm{~K}$.

\section{VALIDATION AND EVALUATION OF NUMERICAL UNCERTAINTIES}

In addition to the classical LES problem of defining inlet boundary conditions that match an experimental facility (assuming that all information is known), ${ }^{53,54}$ LES is subject to several uncertainties. For instance, grid resolution and SGS modeling are two degrees of freedom that can have a strong influence on the numerical predictions. Therefore, as part of the validation process, these numerical uncertainties need to be assessed. In Secs. IV and V, the total physical time used to construct the statistics represents about 17 cycles, if not specified otherwise, where one cyclic period is evaluated from the Strouhal number corresponding to the impinging frequency of the main large scale structures in the simulation $\left(S t_{D}=f D / U_{b} \approx 0.79\right.$ based on velocity and wall heat flux signal analysis). The temporal averaging procedure starts when the flow becomes statistically stationary. Note also that to increase the convergence of the statistics, quantities of interest are averaged in the homogeneous azimuthal direction after temporal averaging. The heat transfer results 


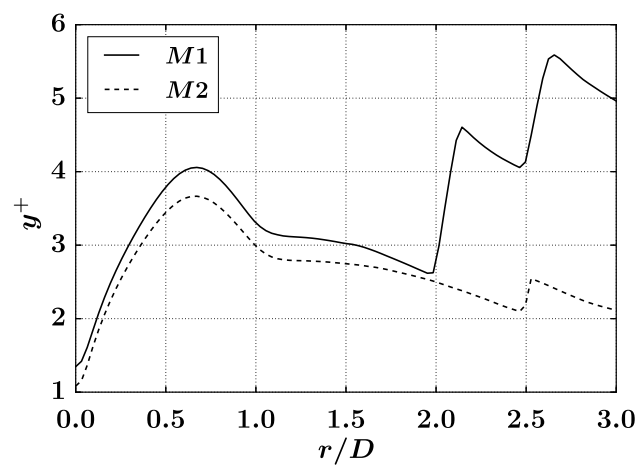

FIG. 2. $y^{+}$on the plate as a function of the distance from the jet axis $r / D$.

are compared hence using the mean Nusselt number defined as

$$
N u(r)=q_{w}(r) D /\left(k_{f}\left(T_{w}-T_{\infty}\right)\right),
$$

where $q_{w}(r)$ is the temporally and azimuthally averaged wall heat flux and $k_{f}$ is the thermal conductivity of air at $T_{\infty}$.

\section{A. Grid resolution}

Two unstructured hybrid grids are considered for this study. They are both composed of tetrahedral cells and ten prism layers on the plate to increase the near-wall resolution without increasing too much the total number of cells. The first mesh, M1, composed of 21 million cells with specific refinement in the free jet, stagnation, and wall jet regions. The normalized near wall distance $y^{+}$ remains below 5 in the region of interest. M2 differs from M1 in the near wall region. Points are added in the wall jet and $y^{+}$remains below 3 except in the region $0.5<r / D<1$ where it reaches a maximum value of 3.5 (see Fig. 2). The spatial resolution in the radial and azimuthal direction is also increased for M2. The main characteristics of the two meshes are summarized in Table I. Mean velocity profiles are presented in Figs. 3 and 4 for both radial and axial components. The experimental data reported by Tummers, Jacobse, and Voorbrood ${ }^{35}$ are also plotted for comparison. First, one can note that a good agreement is found between the LES and the experiment for the mean velocity profiles indicating that LES reproduces correctly the development of the mean wall jet. Discrepancies appear for the axial velocity profile at $r / D=1$ for $y / D>0.3$ (see Fig. 4(b)). This is caused by the co-flow that imposed the value of axial velocity outside the wall jet region while in the experiment, the axial velocity is driven by the natural entrainment of the ambient air. However, inside the wall jet region for $y / D<0.3$, a good agreement is found between LES and experiments, supporting the statement that the co-flow does not influence the wall jet behavior. The profiles of the variances of the axial and radial velocities are presented for two radial locations in Figs. 5 and 6. Discrepancies are found between LES and experiments and there are several possible reasons for these behaviors. First, in the simulation only a pipe of length $2 D$ is used while in

TABLE I. Computational grid properties for the low resolution mesh (M1) and the high resolution mesh (M2).

\begin{tabular}{lcc}
\hline \hline & $\mathrm{M} 1$ & $\mathrm{M} 2$ \\
\hline Number of grid cells (millions) & 21 & 42 \\
Number of prisms (millions) & 4 & 9 \\
$y^{+}[-]$ & $2-5$ & $1-3.5$ \\
$r^{+} \approx r \theta^{+}[-]$ & $8-40$ & $5-20$ \\
$d t[\mu \mathrm{s}]$ & 0.312 & 0.397 \\
$\mathrm{CPU}$ time for 1 cycle $(\mathrm{h})$ & 450 & 710 \\
\hline \hline
\end{tabular}




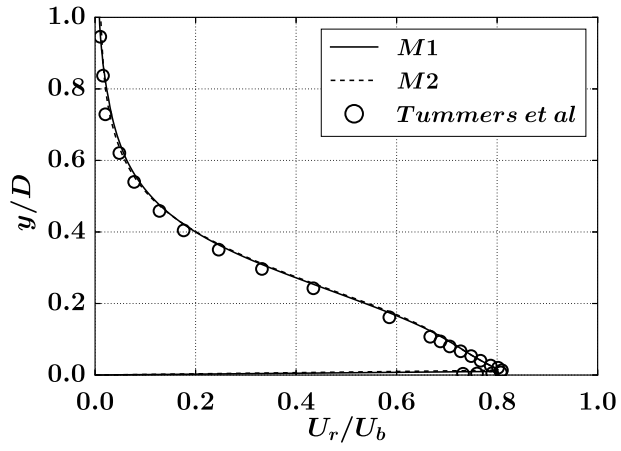

(a)

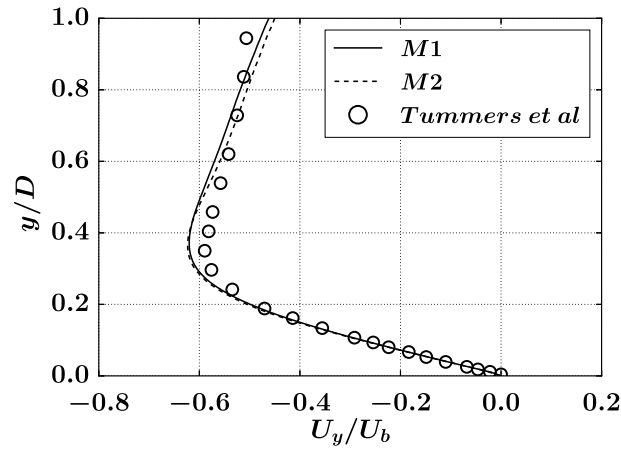

(b)

FIG. 3. Radial mean velocity $U_{r} / U_{b}$ (a) and axial mean velocity $U_{y} / U_{b}$ (b) as a function of the distance from the wall $y / D$ at the radial position $r / D=0.5$. (○): Experiments of Tummers et al., 2011, (-): M1 (see Table I), and (--): M2 (see Table I).

the experiments the pipe is $76 \mathrm{D}$ long. The vortical structures developing in the free jet region are certainly more coherent than in the experiment generating larger velocity fluctuations. Such features were observed by Lodato, Vervisch, and Domingo ${ }^{49}$ for the same configuration and the same SGS model, i.e., WALE. Another explanation proposed by these authors point to the WALE model that does not allow energy backscatter from the unresolved to the resolved fields resulting on average to a too dissipative model and slowing down the process of vortex breakup which is in favor of higher levels of resolved velocity fluctuations. Although such turbulent modeling difficulty is out of scope of the current work, they indicate that their similarity mixed model is potentially capable of taking into account such energy backscatter, improving slightly the predictions of second order moments in the near wall region.

The two grid resolutions used here give similar results except for the axial velocity at $r / D=1$ where differences of $20 \%$ appear on the prediction of the peak of the variance. Aside from that, the global tendency is well predicted by both M1 and M2. Having knowledge of these differences, the radial evolution of the mean radial wall shear stress and the mean Nusselt number is compared in Fig. 7. A slight improvement of the prediction of the plateau near $r / D=1.5$ in the wall shear stress distribution is noticed with M2. However, for the Nusselt number, the two curves are superimposed except for $r / D>2.5$ where the coarsening of the mesh is stronger for M1 than for M2. It can be concluded that the mesh resolution has a negligible effect on wall quantities despite the discrepancies observed for the variance of the axial velocity.

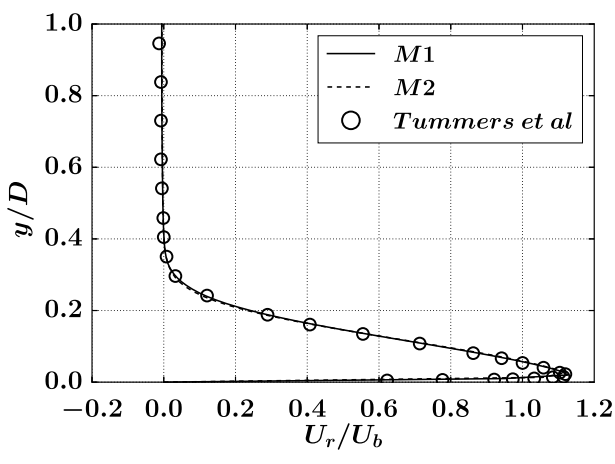

(a)

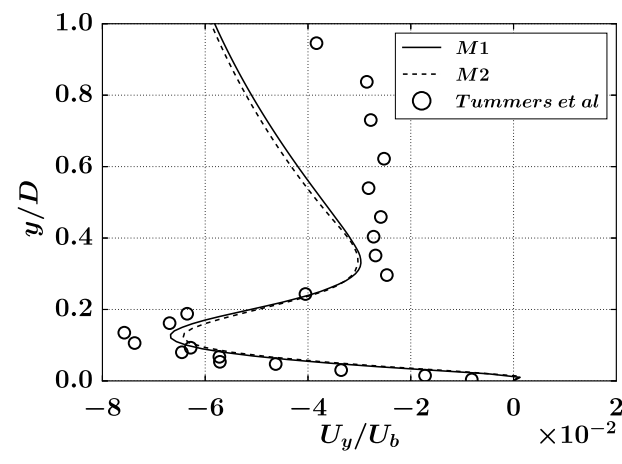

(b)

FIG. 4. Radial mean velocity $U_{r} / U_{b}$ (a) and axial mean velocity $U_{y} / U_{b}$ (b) as a function of the distance from the wall $y / D$ at the radial position $r / D=1$. ( $)$ : Experiments of Tummers et al., 2011, (-): M1 (see Table I), and (--): M2 (see Table I). 


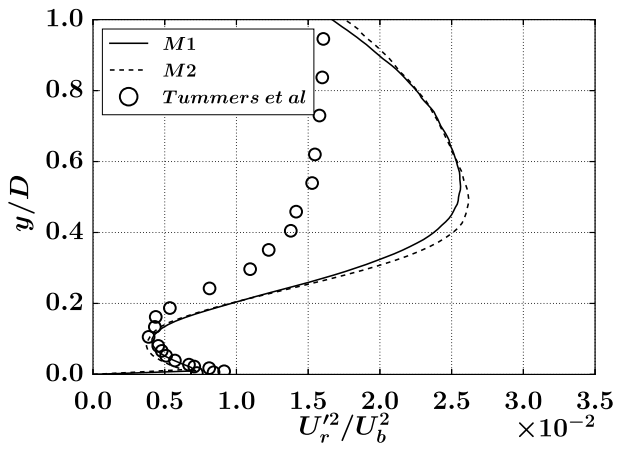

(a)

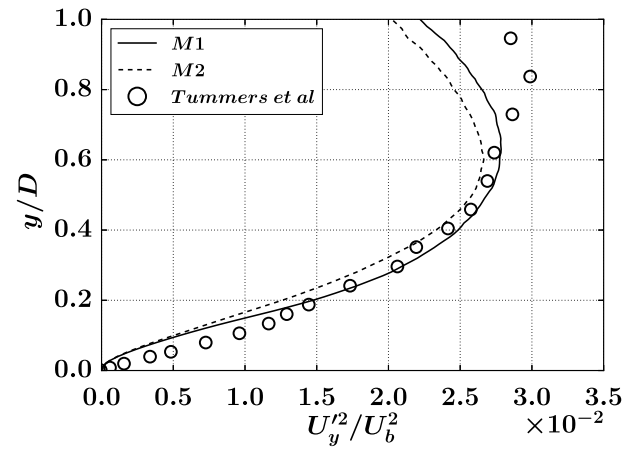

(b)

FIG. 5. Variance of the radial velocity $U_{r}^{\prime 2} / U_{b}^{2}$ (a) and axial velocity $U_{y}^{\prime 2} / U_{b}^{2}$ (b) as a function of the distance from the wall $y / D$ at the radial position $r / D=0.5$. (०): Experiments of Tummers et al., 2011, (-): M1 (see Table I), and (--): M2 (see Table I).

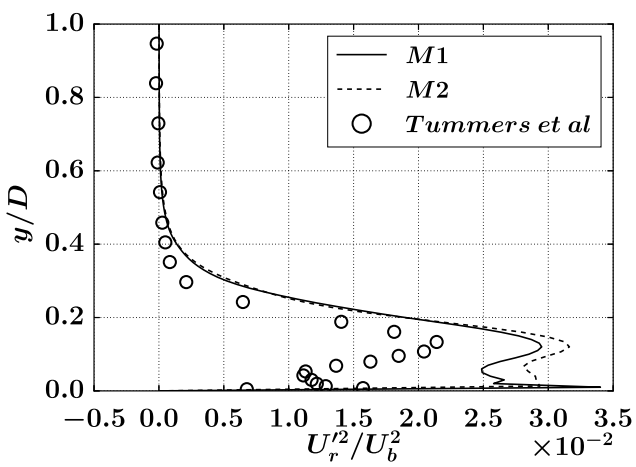

(a)

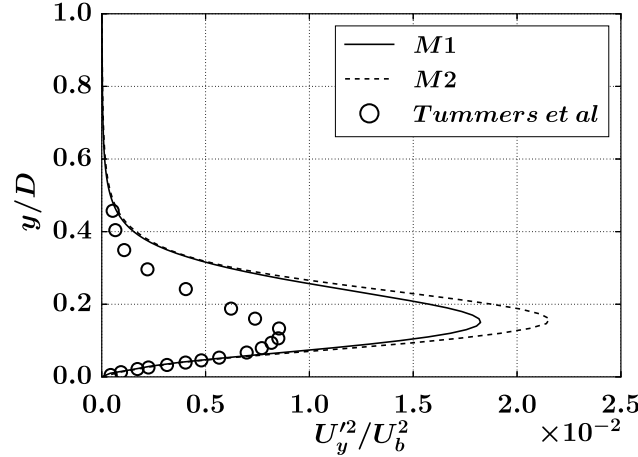

(b)

FIG. 6. Variance of the radial velocity $U_{r}^{\prime 2} / U_{b}^{2}$ (a) and axial velocity $U_{y}^{\prime 2} / U_{b}^{2}$ (b) as a function of the distance from the wall $y / D$ at the radial position $r / D=1$. (O): Experiments of Tummers et al., 2011, (-): M1 (see Table I), and (--): M2 (see Table I).

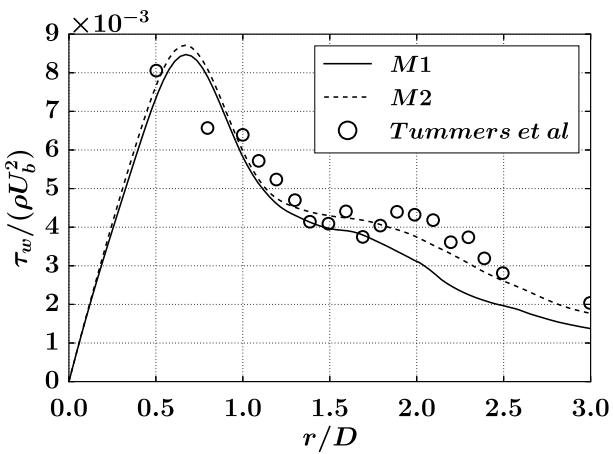

(a)

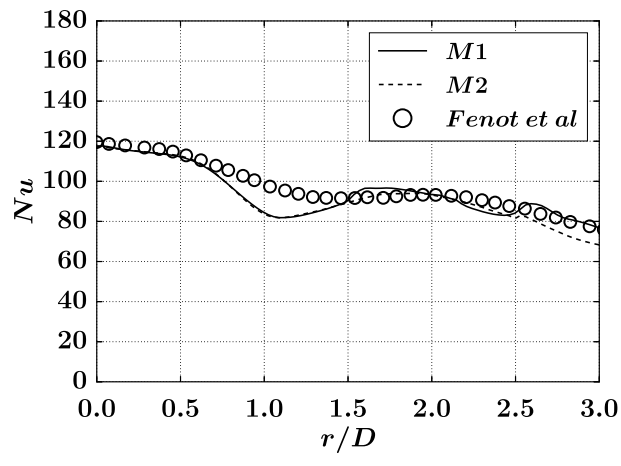

(b)

FIG. 7. Mean wall shear stress $\tau_{w} /\left(\rho U_{b}^{2}\right)$ (a) and mean Nusselt number $N u$ (b) as a function of the distance from the jet axis $r / D$. (O): Experimental data, (-): M1 (see Table I), and (--): M2 (see Table I).

\section{B. Subgrid-scale model}

In this section the impact of the SGS model on the velocity profiles and the wall quantities is assessed. Two SGS models are compared with mesh M2. The first one is the WALE model ${ }^{46}$ based 


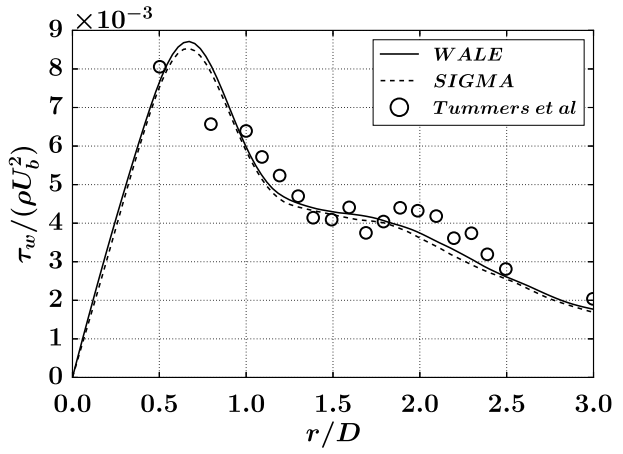

(a)

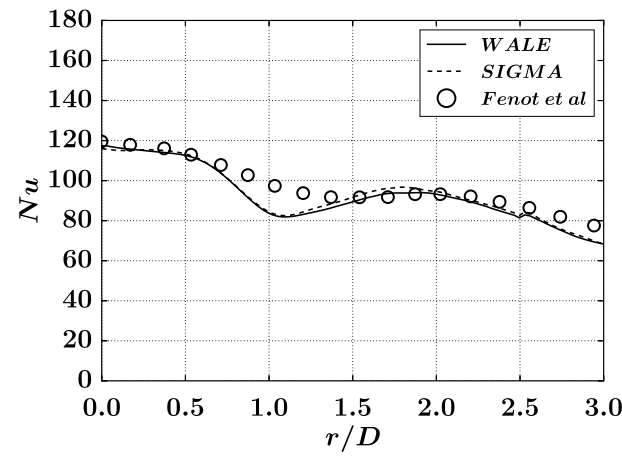

(b)

FIG. 8. Mean wall shear stress $\tau_{w} /\left(\rho U_{b}^{2}\right)$ (a) and mean Nusselt number $N u$ (b) as a function of the distance from the jet axis $r / D$. (O): Experimental data, (-): WALE SGS model, and (--): SIGMA SGS model.

on the second invariant of the traceless symmetric part of the square of the velocity gradient tensor. This model is built to produce a zero turbulent viscosity in the case of a pure shear (important to handle transitional flow) and to recover the proper near-wall scaling of the turbulent viscosity. It was used for the grid convergence study of Sec. IV A. The second model tested here is the $\sigma$ model based on the singular values of the velocity gradient tensor. ${ }^{55}$ It is designed to produce a zero turbulent viscosity for any two-dimensional and/or two-component flows and when the resolved scales are either in pure axisymmetric or isotropic expansion/contraction. Moreover, it recovers the proper near wall scaling of the turbulent viscosity. For sake of brevity, the velocity profiles are not presented here; however, a good agreement is found between the two models for the mean and RMS velocity profiles. Maximum differences of about $10 \%$ appear for the profiles of the variance of the radial and axial velocity components. Finally, wall quantities are found not to be affected by the change of the SGS model (see Fig. 8).

The sensitivity analysis of the results to grid resolution and to SGS model highlight that as for experiments, numerical simulations are subject to uncertainties. Nevertheless, in that case, these uncertainties affect mainly the levels of the variance of the velocity field but do not impact wall heat transfer. The physics that produces the non-monotonic variation of the radial distribution of the Nusselt number seems furthermore well captured by LES. Therefore, this fully unsteady LES database can be used to characterize and improve our understanding of the physics behind the existence of the double peak of such a flow. For all the following, the LES predictions obtained with mesh M2 and the SGS model WALE are used.

\section{RESULTS AND DISCUSSIONS}

As LES gives access to the full 3D time-dependent flow field, the main idea is here to use this space-time information to provide additional details on the main mechanisms responsible for the non-monotonic behaviour of the radial Nusselt distribution. First, a quick description of the statistical tools used for the analysis is provided. The flow dynamics is then analyzed in terms of large scale organization and near-wall aerothermal behavior. Finally the heat transfer at the wall is described and connected to the flow dynamics.

\section{A. Statistical tools}

Based on the one point temporal evolution of a variable $x(t)$, the common quantities that can be extracted are the first order moment (i.e., mean $\langle x(t)\rangle)$ and the second central moment (i.e., variance $\left.\left\langle(x(t)-\langle x(t)\rangle)^{2}\right\rangle\right)$. Dimensionless higher order central moments, such as Skewness (Sk) and Kurtosis $(\mathrm{Ku})$, are also useful when one wants to have a better idea of the PDF's shape ${ }^{56}$ or in the presence of intermittent events as they are more sensitive to intermittency. Sk and Ku are, respectively, the 
third and fourth dimensionless central moments. The skewness evaluates the balance between the left and the right parts (with respect to the mean value) of a PDF. The skewness of a symmetric PDF, like Gaussian distributions, is 0 but the reverse is not true. For unimodal distributions, i.e., single peak, the skewness can also be seen as the "distance" from the mean to the peak, i.e., the mode (or most likely value). Positive (respectively, negative) skewness implies that the mean value is greater (respectively, lower) than the mode value. The interpretation of kurtosis is more difficult ${ }^{57}$ but can be viewed as a movement of mass that does not affect the variance. ${ }^{58}$ High kurtosis value implies that, for the same distribution, mass is moved simultaneously from the shoulder to the tails and to the mean value of the distribution to leave the variance unchanged. This results in an increase of the probability of occurrence of values very close to the mean and extreme values located at the tails. For small kurtosis, the mass is moved from the mean and the tails to the shoulder of the distribution and in the symmetric case the distribution is flattened. One may notice that the kurtosis value is influenced by the asymmetry of the distribution. ${ }^{59}$ Recall for reference that the kurtosis of a Gaussian PDF is $K u=3$. For a more detailed description of the meaning of these two quantities in terms of PDF and sample set, the reader is referred to Ref. 60.

\section{B. Flow dynamics}

In this section the large scale organization is described and compared to the available literature. High order statistics are used to go further in the characterization of the dynamics and PDFs are built to highlight the influence of the large scale structures on the near-wall region.

\section{Large scale organization}

As mentioned in the Introduction, there are two kinds of large scale structures present in impinging jet flows. The primary vortices are formed in the free jet region due to the initial instability of the shear layer while the secondary vortices are developing near the wall due to the interaction of the primary vortices with the wall (see Fig. 9). The presence of the primary and secondary vortices is evidenced here using instantaneous iso-surfaces of Q-criterion colored by the sign of the azimuthal vorticity as proposed by Dairay et al. ${ }^{21}$ and shown in Fig. 10. The negative azimuthal vorticity, in black here, characterizes the primary vortices while the secondary structures, located closer to the wall, are identified by positive azimuthal vorticity. Figure 11 shows the space/frequency map of the pressure fluctuation amplitude constructed using 19 probes located along the shear layer at $r / D=0.5$ at the azimuthal position $\theta=0$. The space resolution is $\Delta(y / D)=0.1$ and the frequency resolution is $\Delta\left(S t_{D}\right)=0.06$. Different peaks are observable along the shear layer. These peaks correspond to $S t_{D}=1.6,1.13,0.95,0.8,0.6$. It is interesting to note that the dominant frequency decreases as the probe approaches the plate (positioned at $y / D=0$ ) without reaching the "free jet preferred mode," $S t_{D}=0.3$, due to the small nozzle to

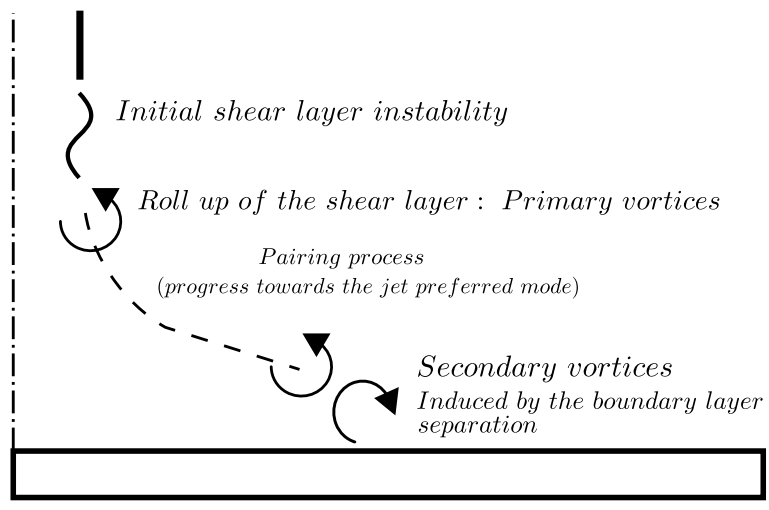

FIG. 9. Description of the large scale structures present in impinging jet flows. 


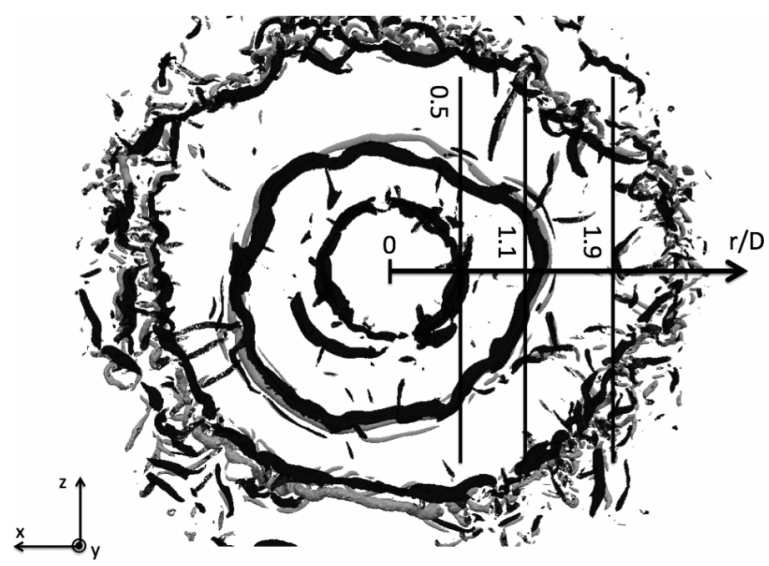

FIG. 10. Instantaneous iso-surface of Q-criterion colored by the azimuthal vorticity sign: positive azimuthal vorticity (grey) and negative azimuthal vorticity (black).

plate distance. Frequency halving occurs as the vortices approach the wall due to vortex pairing 9 except for $S t_{D}=0.95$ which could be the results of interaction between mode $S t_{D}=1.13$ and mode $S t_{D}=0.8$. Finally close to the wall, two peaks are still observable at $S t_{D}=0.6$ and $S t_{D}=0.8$. Note that the spatial extension of the peaks is in part linked to the spatial intermittency of the vortex pairing location, i.e., vortices are not always merging at the same axial location for such a flow, indicating that the observed coherent structures are neither fully deterministic nor stochastic. The deterministic part is linked to the generation of the coherent structures, assuming that the phenomenon is periodic with a well defined period that is a good approximation if a feedback loop exists as for high-speed subsonic jet ${ }^{61}$ or if an external forcing is present. The stochastic part however relates to the interaction between vortical structures.

One of the fundamental problems behind impinging jets is the impingement of vortex ring for which the generation of secondary and tertiary vortices is observed as well as a rebound of the primary vortices. ${ }^{12}$ In the context of impinging jet flow at high Reynolds number, the rebound of the primary vortices is difficult to detect as it is part of a transitional flow. This is why high order statistics that are more sensitive to intermittent events, such as those generated by unsteady coherent structures, are preferred for this study. Using the fact that vortices generate negative spikes in the temporal distribution of pressure fluctuations, the rebound of the primary vortices is highlighted using the azimuthally averaged skewness issued by the analysis of the temporal pressure evolution. Note that

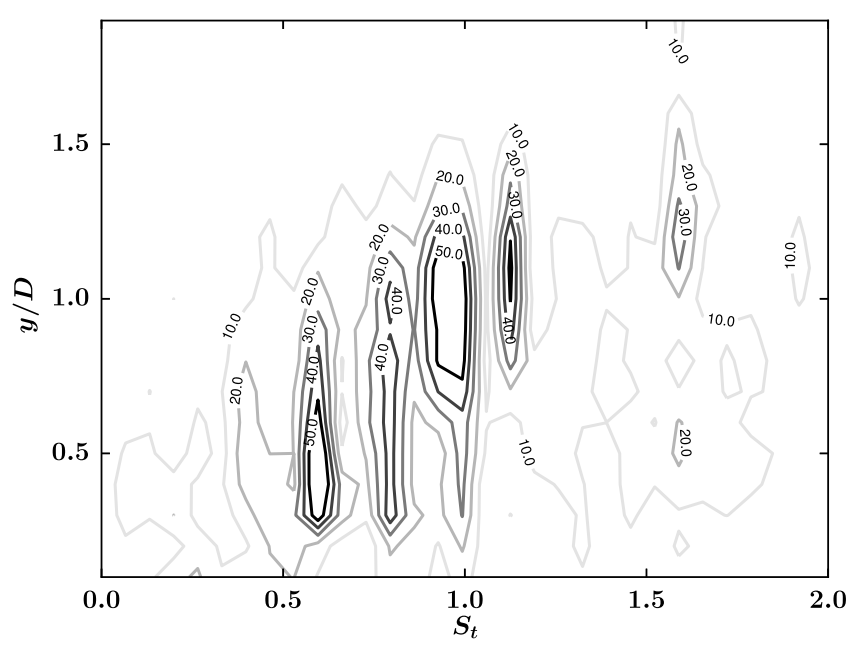

FIG. 11. Iso-contours of pressure fluctuation amplitude $(P a)$ in the $\left(y / D, S t_{D}\right)$ plane at $r / D=0.5$ and $\theta=0$. 
these diagnostics are preferred to classical autocorrelation length scales due to several reasons. First, using autocorrelation length scales implies to choose an a priori accurate definition of the researched time scale event. As such, an arbitrary threshold for the integration of the auto-correlation function, for example, to yield a measure of the structure time scale, will produce uncontrolled results and a potential misinterpretation of the results. Reconstruction of the length scale, based on one-point autocorrelation functions, requires the use of Taylor's hypothesis, the validity of which is in doubt in presence of large scale interactions ${ }^{62}$ For wall bounded flow, it has also been shown that the accuracy of the Taylor's hypothesis decreases with the increase of the projection distance. ${ }^{63}$ Such a hypothesis and arbitrary threshold are not required for the skewness as the negative skewness is induced by the passage of the vortical structures in this case. Note finally that computing autocorrelation functions imposes to store information which can rapidly increase memory requirement while the computation of statistics can be done during the simulation keeping only the final value. As expected the skewness of the temporal distribution of pressure, plotted in the $(y / D, r / D)$ plane in Fig. 12, is negative (indicative of the presence of events happening for values below the mean) in the shear layer of the free jet region $(r / D \approx 0.5$ and $y / D>0.5)$ as the passage of the primary vortices induces negative pressure fluctuations in the temporal signal. Two main regions of remarkable values for skewness of pressure appear near the wall. The intermittent formation and convection of secondary vortices generate region B where $S k<-1.6$. This region disappears near $r / D=1.9$. Note that this region B coincides with the location of initiation of the unsteady boundary layer flow separation that occurs just before the formation of secondary vortices ${ }^{14}$ at $r / D \approx 1$. Region A relates to the primary vortices which orient upwards as the radial location increases. This reveals the rebound of the primary vortices which is induced by the secondary vortices velocity field. ${ }^{12}$ The rebound starts here at $r / D=1.6$ and disappears near $r / D=2$ (extent of region $\mathrm{A}$ ).

To complement the analysis of the flow dynamics, the near wall behavior of the temperature and the velocity fields are analyzed to investigate the effect of the vortical structures on the aerothermal field.

\section{Temperature field}

The near wall thermal behavior of the flow is analyzed using numerical probes radially distributed at a normal distance $y / D=0.01$ above the plate. Based on the analysis of several instantaneous snapshots (not shown here), at this normal distance from the plate, the probes are found to mainly reflect the effect of the secondary structures on the near wall flow. For the discussed diagnostics, 12 probes are distributed in each of 10 azimuthal directions as shown by Fig. 13. Sampling is made for 17 cycles, where one cycle is evaluated from the impinging frequency of the main large scale structures, and 33000 samples are collected for each probe. Note that this leads to

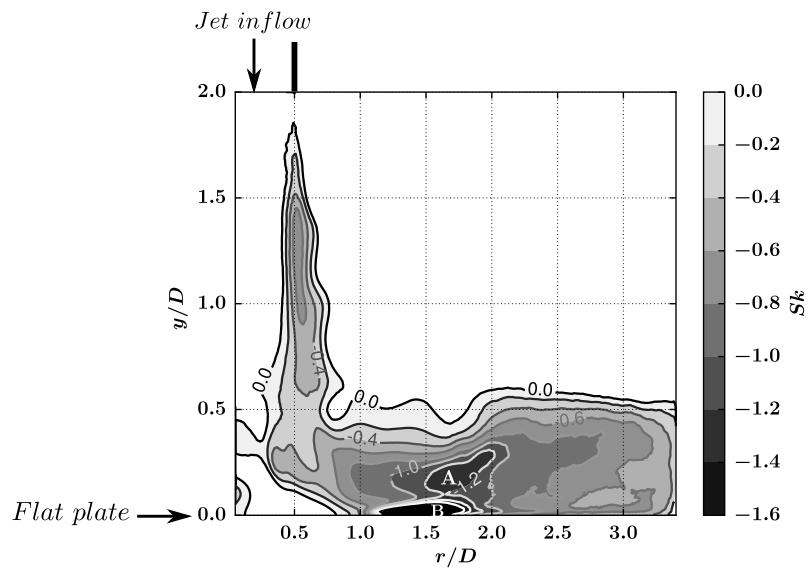

FIG. 12. Iso-contours of azimuthal averaged skewness of the pressure temporal distribution in the $(y / D, r / D)$ plane. 


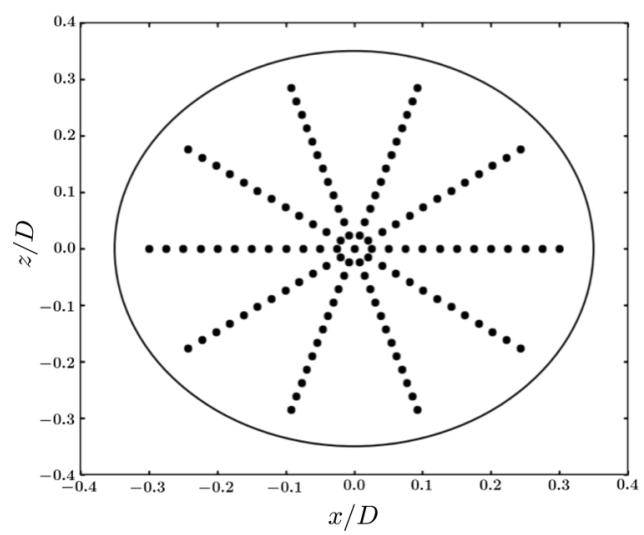

FIG. 13. Location of the numerical probes in the $(z / D, x / D)$ plane used for the PDF analysis. The probes are located at $y / D=0.01$ for the near wall investigation and at $y / D=0$ for the analysis of the wall heat flux.

a sampling frequency which is in agreement with the Nyquist frequency for the phenomena investigated here. Based on these samples the PDFs are estimated using histograms that are normalized to respect the property: $\int_{-\infty}^{+\infty} P D F(x) \mathrm{dx}=1$. The temperature PDF are presented in Fig. 14 for 9 radial locations for the normalized temperature defined as $T^{*}=\left(T-T_{\infty}\right) /\left(T_{w}-T_{\infty}\right)$, where $T$ is the local instantaneous temperature. These PDFs are close to Gaussian distribution for $r / D=0.5$ and $r / D=0.75$. Deviation from a Gaussian starts at $r / D=1$ where the PDF gets positively skewed. This positive skewness indicates that the near wall fluid is considerably heated by the plate. It is believed that this is the consequence of the intermittent local flow deceleration which results in a local increase of the fluid residence time, due to the adverse pressure gradient generated by the approach of the primary vortices. This phenomenon takes place before the separation of the boundary layer and seems amplified by the boundary layer separation. When the boundary layer is separated, i.e., around $r / D \approx 1.1$ (cf. Fig. 12), the secondary vortex is initiated amplifying the segregation between hot and cold fluids near the wall, thereby explaining the bimodal shaped PDFs at these locations, i.e., $1.25 \leq r / D \leq 1.75$. The first PDF peak, located on the left side of the mean value, is a cold mode and has the highest probability while the second peak, located on the right side of the mean value, is a hot mode with a lower probability. One can also note that this region
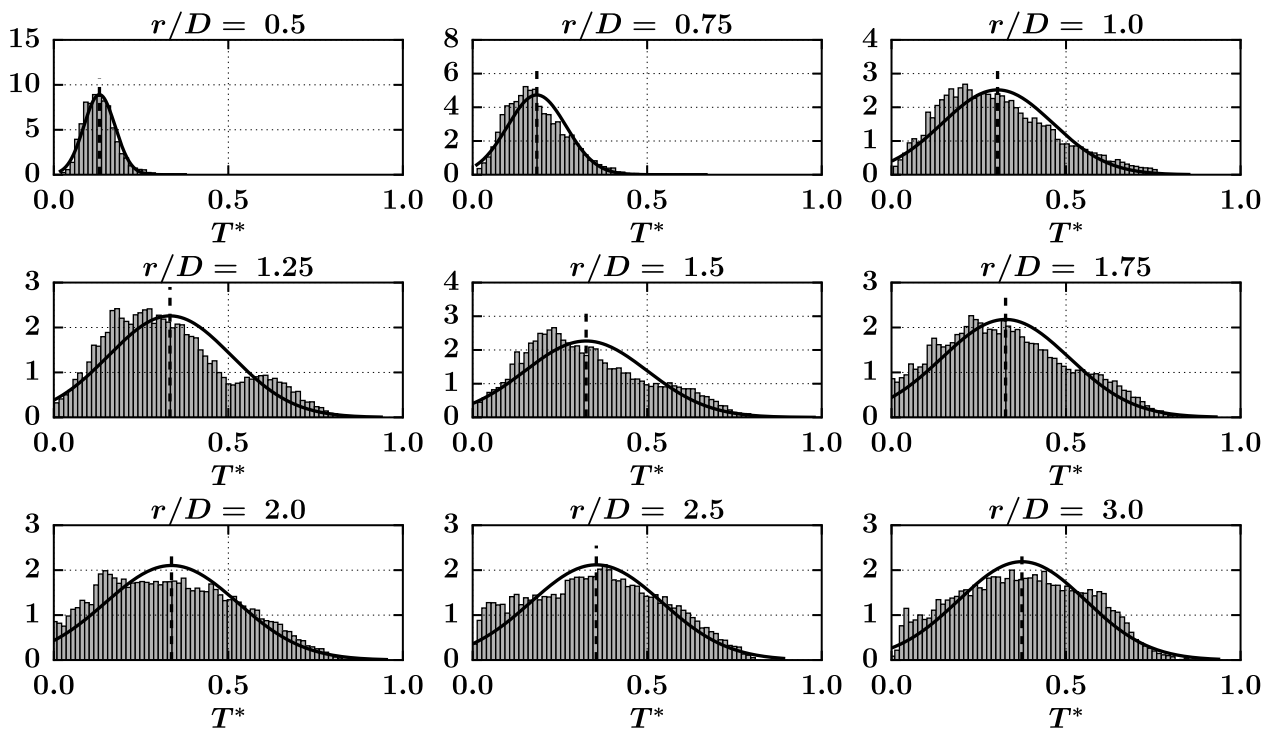

FIG. 14. PDFs of the dimensionless temperature time series $T^{*}$ at 9 locations along the radius. (-): Gaussian distribution with the same mean and standard deviation values as the temperature PDFs, (--): mean value. 
of bimodal behavior corresponds to the radial extent of region B previously linked to the secondary vortices. For $r / D \geq 2$, the distributions are flatter indicating a less organized mixing. As the probes are located very close to the wall, it is most likely that the hot mode is induced by the recirculating fluid that is trapped and heated by the plate as observed by Hadžiabdić and Hanjalić, ${ }^{22}$ while the cold mode is the consequence of a local cold fluid intrusion in the wall boundary layer flow induced by the secondary vortices.

The numerical approach used in this study allows to record simultaneously the temperature and the velocity time series and such samples are used in Sec. V B 3 to gain further insight into the near-wall mixing process.

\section{Joint velocity-temperature analysis}

Several authors have qualified the above mentioned process as "cold fluid flux" towards the wall to explain the mean heat transfer enhancement. ${ }^{21,32}$ However to the authors' best knowledge, this claim has not been proven except by Dairay et al. ${ }^{21}$ who used conditional averaging near the location of a cold spot. In this study, the problem is addressed using joint axial velocity-temperature PDFs that do not require any arbitrary threshold value. Indeed, depending on whether the observation point is upstream or downstream a vortical structure, the 2 possible events are the fluid injection or the fluid ejection, i.e., negative or positive axial velocity (see Fig. 15). Adding the temperature to the analysis allows therefore to make the distinction between hot and cold events (hot and cold being defined with respect to the local time and azimuthal averaged temperature). It is then possible to construct the 4 quadrants corresponding to the 4 possible events, i.e., cold/hot fluid ejection/injection as shown in Fig. 16. The corresponding joint axial velocity-temperature PDFs are shown in Fig. 17 for 8 radial locations. Close to the nozzle, i.e., $r / D \leq 0.75$ the dominant event is the fluid injection as the flow is under the influence of the axial jet. Relatively far away from the nozzle, i.e., $r / D>1$, one can note that there is still a clear organization of the mixing until $r / D=1.75$ with the cold fluid injection being the dominant process. This is in agreement with the observation made on the temperature PDF where the cold mode appears to be the most likely. It is then possible to state that the large scale structures generate preferentially a cold fluid flux towards the wall. A more quantitative representation is presented in Fig. 18 where the joint PDFs are integrated over each quadrant to compute the probability related to each of the 4 possible events. Again it appears clearly that the dominant process in the region of formation and convection of the secondary vortices is the cold fluid injection. After the rebound of the primary vortices however, the cold fluid injection and hot fluid ejection events have a similar probability.

This section has focused on the flow dynamics highlighting the presence of vortical structures, their effect on the near wall behavior and the rebound of the primary vortices from the wall. The idea is now to compare these results to the wall heat transfer process and make the connection between the large scale organization and the mean heat transfer distribution.

\section{Wall heat transfer}

The first part of this section focuses on the unsteady behavior of the wall heat transfer process using instantaneous visualizations and temporal maps. Then, the statistical tools are used to confirm in a statistical sense the instantaneous behavior and connect the results to the flow dynamics.

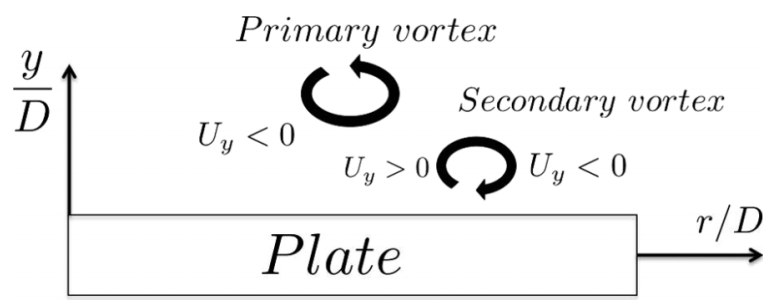

FIG. 15. Large scale organization of the flow near the wall. 


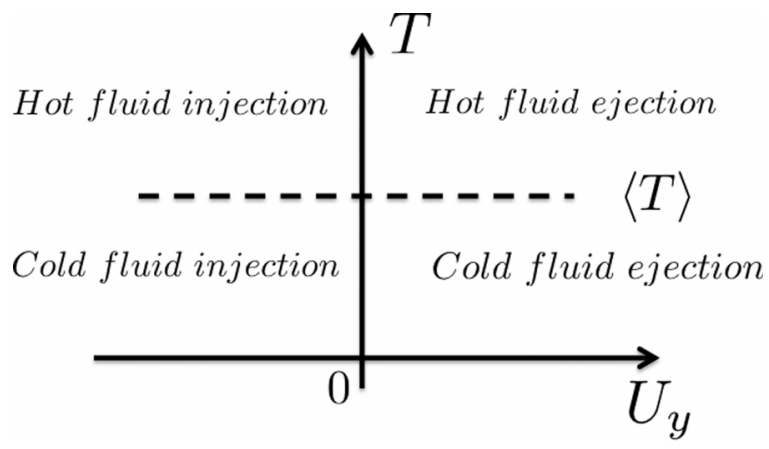

FIG. 16. Representation of the 4 quadrants in the $\left(U_{y}, T\right)$ space. Injection and ejection events are identified respectively by $U_{y}<0$ and $U_{y}>0$. Hot and cold events are identified respectively by $T>\langle T\rangle$ and $T\langle\langle T\rangle$, where $\langle T\rangle$ is the time and azimuthal averaged local temperature.

\section{Unsteady behavior}

The instantaneous evolution of the fluctuating Nusselt number for 2 distinct instants is presented in Fig. 19. To ease the analysis, a radial axis is added recalling that the location of the local minimum is at $r / D=1.1$ while the secondary maximum appears at $r / D=1.9$. For both instants, two cold fronts, i.e., region of positive Nusselt number fluctuations, identified as cold front $\mathrm{A}$ and $\mathrm{B}$ are visible. The cold front $\mathrm{B}$ is stronger and has a better azimuthal coherence than cold front $\mathrm{A}$. Cold front $\mathrm{B}$ is generated by the secondary vortex that is located closer to the wall than the primary vortex. Note also that cold front B loses its azimuthal coherence near the location of the secondary
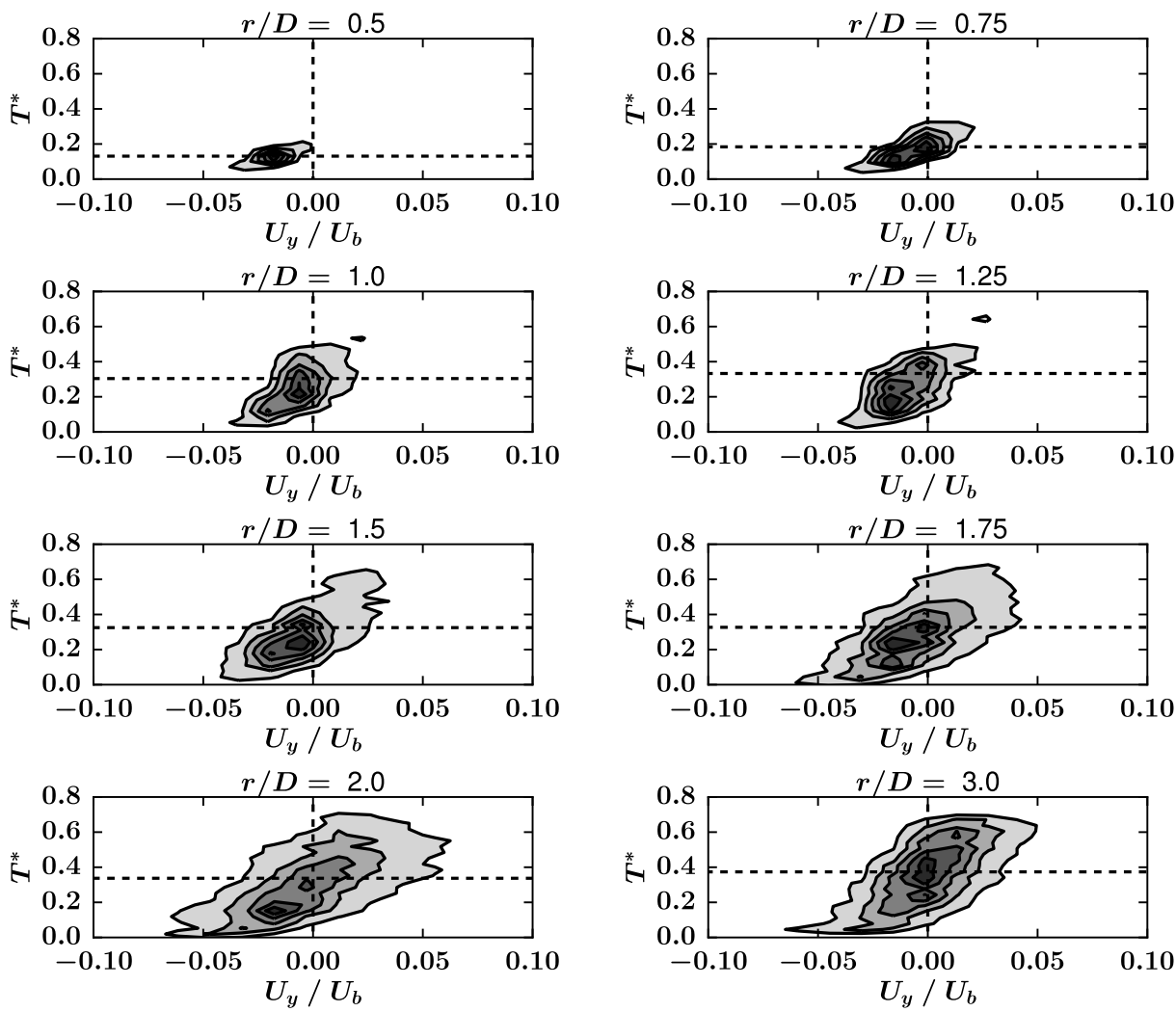

FIG. 17. Joint probability distribution, $P\left(U_{y}, T\right)$, at $y / D=0.01$ for 8 radial locations. (--): Limits of the 4 quadrants; scale: high probability (black) and zero probability (white). 


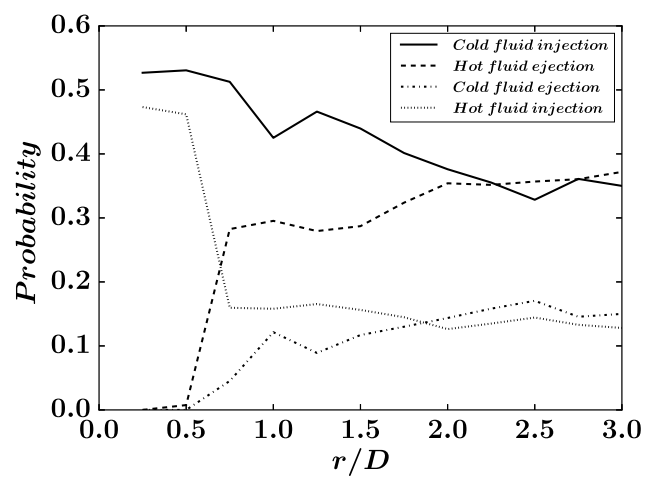

FIG. 18. Probability of the 4 possible events, i.e., cold fluid injection/ejection and hot fluid injection/ejection, as a function of the radial distance from the jet axis $r / D$.

peak at $r / D=1.9$. Fig. 20 shows the spatio-temporal maps of the Nusselt number extracted from numerical probes placed on the plate at $\theta=0$, i.e., along the $\mathrm{r}$ axis with $t^{*}=t S t_{D} U_{b} / D$ is the dimensionless time allowing to track the temporal evolution of the cold fronts. Cold front $\mathrm{B}$ is generated at $r / D \approx 1$ coinciding with the local minimum in the mean Nusselt radial distribution. Cold front A develops continuously from the stagnation region to $r / D \approx 1.6$ and is seen to accelerate for $0.5<r / D<1$. Then it is slowed down by cold front $\mathrm{B}$, around $r / D \approx 1$, both having approximately the same convection velocity, $U_{c o n v}=0.61 U_{b}$. It seems therefore that the primary vortices are not the only cause behind the generation of cold front $\mathrm{A}$.

The cold air injection induced by the secondary vortex, located just above cold front $\mathrm{B}$, is clearly seen in Fig. 21 where the instantaneous temperature fluctuations along with the streamlines are represented in a wall-normal plane at $\theta=0$. This feature is in agreement with the observation by Dairay et al. ${ }^{21}$ where secondary vortex is detected close to cold spots. The hot front upstream of cold front $\mathrm{B}$ is the consequence of the trapped fluid in the recirculation region issued by the

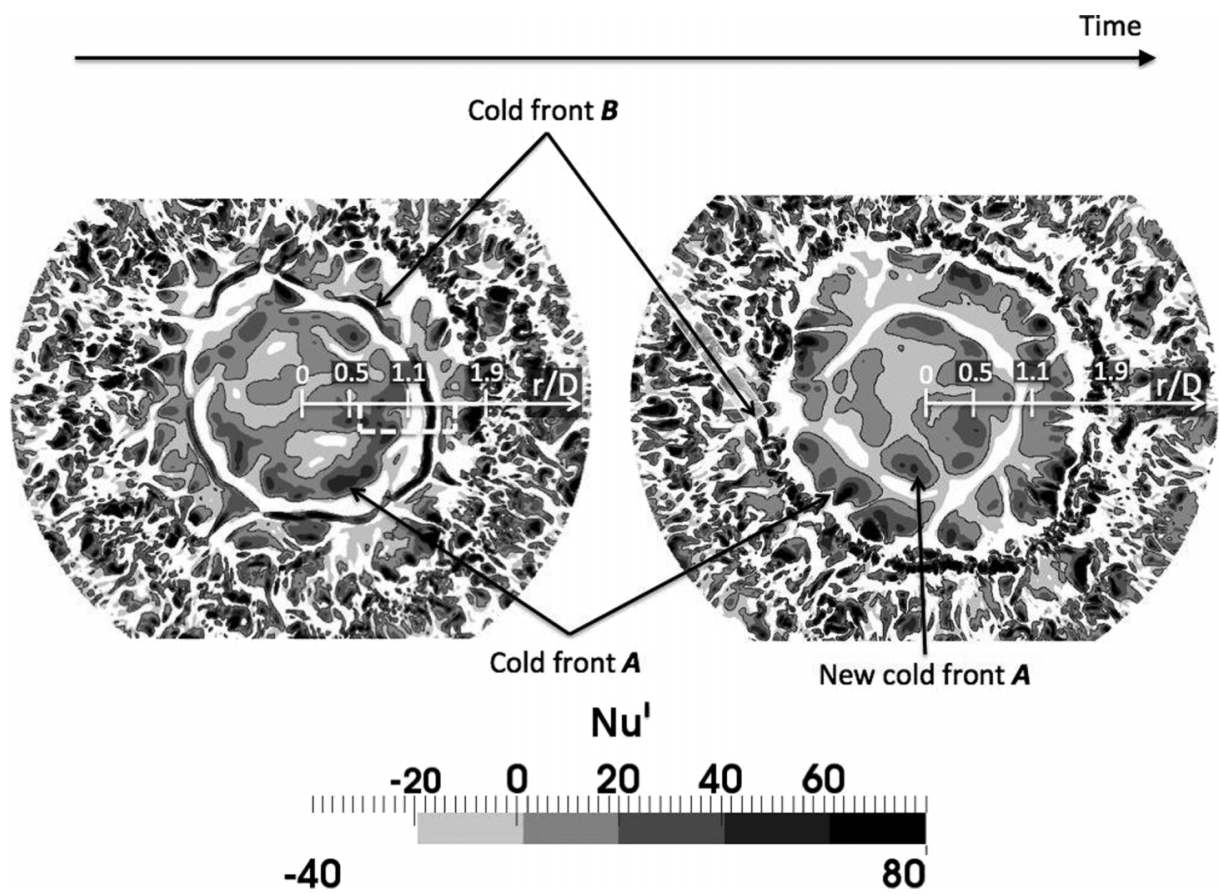

FIG. 19. Sequence of 2 instantaneous snapshots of the local fluctuating Nusselt number, $N u^{\prime}$, on the plate identifying two cold fronts related to the passage of the large scale coherent structures. (-): Isocontours of $N u^{\prime}=0$. 


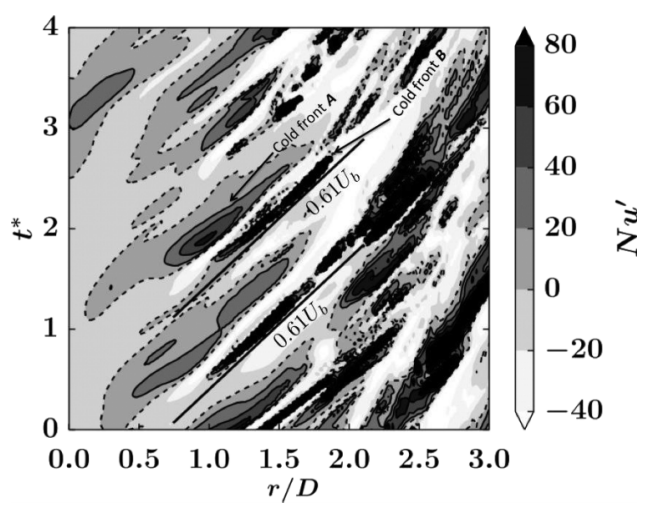

FIG. 20. Contours of the Nusselt number fluctuations, $N u^{\prime}$, at $\theta=0$ along the $\mathrm{r}$ axis in the $\left(t^{*}, r / D\right)$ plane. Straight black solid lines: Convection velocity $U_{\text {conv }}=0.61 U_{b}$. (--): Isocontours of $N u^{\prime}=0$.

boundary layer separation confirming the previous analysis based on the temperature PDFs. It also appears that the positive Nusselt fluctuation for cold front B is approximately two times higher than for cold front A as evidenced by the lower part of Fig. 21 which pictures the $N u^{\prime}$ map on the wall surface near the normal plane.

Cold front $\mathrm{A}$ is located between two primary structures where negative temperature fluctuations are observed near the wall. It is believed that cold front A is mainly the consequence of the unsteady development of the wall jet when not perturbed by the primary vortices. There may be an influence of the primary vortices as some high heat transfer regions localized in space are observed in cold front A. It could be the consequence of an azimuthal instability, as observed for free jets. ${ }^{9}$ Here the plate is placed so close to the jet exit that the transition is incomplete when the primary vortices reach the plate. The azimuthal instability causes the primary structure to be closer to the wall at specific azimuthal locations inducing additional cold fluid intrusions in the boundary layer and localized high heat transfer regions in cold front A.

From this instantaneous analysis, it is concluded that the secondary vortices are linked to one intense cold front that is suspected to generate the strongest thermal events responsible for the occurrence of the secondary peak. The next part focuses on the impact of these cold fronts on

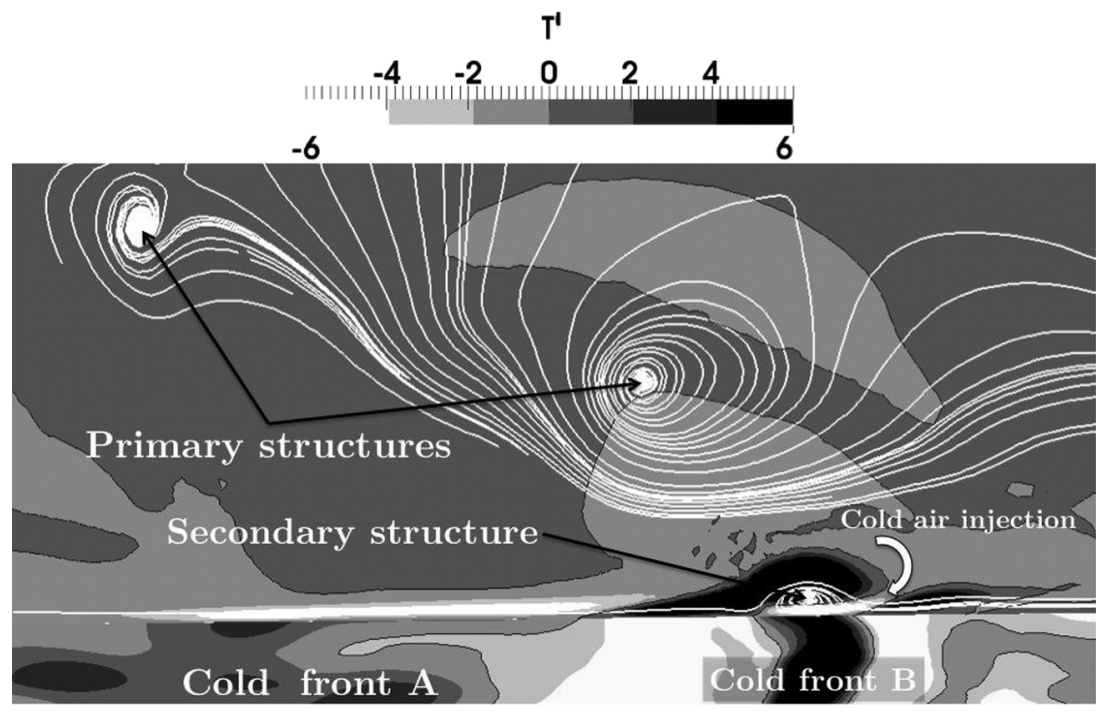

FIG. 21. Instantaneous snapshot of the Nusselt fluctuations, $N u^{\prime}$, on the wall (lower part of the figure) and streamlines along with the temperature fluctuation, $T^{\prime}$, projected in the plane $\theta=0$. The scale for $N u^{\prime}$ is the same as the one used for Fig. 19. $(-)$ : Isocontours of $N u^{\prime}=0$ and $T^{\prime}=0$. 


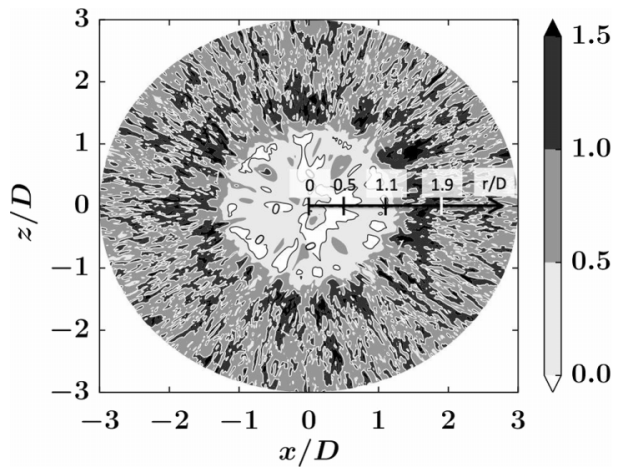

(a)

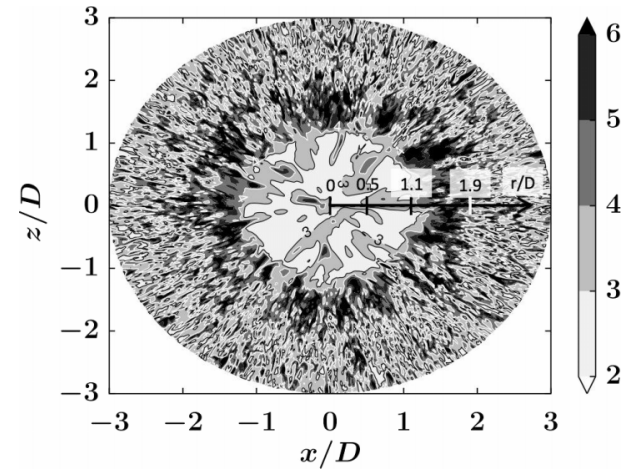

(b)

FIG. 22. Skewness (a) and kurtosis (b) of the Nusselt, $N u$, temporal distribution on the plate in the $(x / D, z / D)$ plane. Black isocontours: $S k=0, K u=3$. White isocontours: $S k=1, K u=4$.

the wall heat transfer temporal distribution to highlight their role in the heat transfer enhancement process.

\section{Statistical analysis}

Skewness and kurtosis of the temporal evolution of the local Nusselt number are in this section constructed at each computational node on the plate to produce spatial maps as presented in Fig. 22. For both quantities, three regions appear which are linked to the different behaviors of the mean Nusselt radial distribution. The first one extends from the stagnation point to the local minimum at $r / D=1.1$. In this region $S k$ and $K u$ values are close to the Gaussian values, i.e., $S k=0$ and $K u=3$. Skewness and kurtosis values start to deviate from the Gaussian values with $S k>1$ and $K u>4$ in the region of heat transfer enhancement $1.1<r / D<1.9$. The positive skewness indicates that relatively strong events that are not balanced by equivalent weak events occur. Relatively high kurtosis values highlight the intermittency of these strong thermal events. After the secondary peak, i.e., $r / D>1.9$, the kurtosis and skewness decrease slowly without returning to the Gaussian values.

The PDFs of the Nusselt number are analyzed using the numerical probes used for the temperature field analysis placed on the plate at $y / D=0$ and with the same sampling. The PDFs are presented at 8 radial locations in Fig. 23. As anticipated, the PDFs are close to the Gaussian shape for $r / D=0$ to $r / D=1$. Afterwards, the shape of the PDFs starts to deviate from Gaussian with an increasing probability of occurrence of relatively low Nusselt number values around $r / D=1.25$, breaking the balance between low and high Nusselt values. This imbalance is to be linked to the unsteady separation of the boundary layer. Then, relatively strong events, i.e., high Nusselt number values, appear and the PDFs skew to the right. This feature explains the observed positive values of skewness previously reported for $1.1<r / D<1.9$ (see Fig. 22(a)). The PDFs remain then skewed up to $r / D=3$ indicating a persistence of relatively strong thermal events that are no longer able to increase heat transfer. After $r / D=2$, the probability to have Nusselt number values smaller than the mean value increases while there are fewer strong events and the probability to have Nusselt number values higher than the mean decreases. This explains why the Nusselt number is decreasing for $r / D>2$ despite the positive skewness. To confirm that the strong thermal events are responsible for the occurrence of the secondary peak, the decomposition of the PDFs in a symmetric and an asymmetric part proposed by Dairay et al. ${ }^{21}$ is used. The important point here is to note that the asymmetric part causes the deviation of the mean value from the mode value. With such positively skewed PDFs, the mean value is greater than the mode value. To assess the importance of the strong intermittent thermal events in the generation of the secondary peak, the mode value is compared to the mean value in Fig. 24. The secondary peak is not recovered in the radial distribution of the mode Nusselt value that is in agreement with the previous results obtained by Dairay et al. ${ }^{21}$ This 

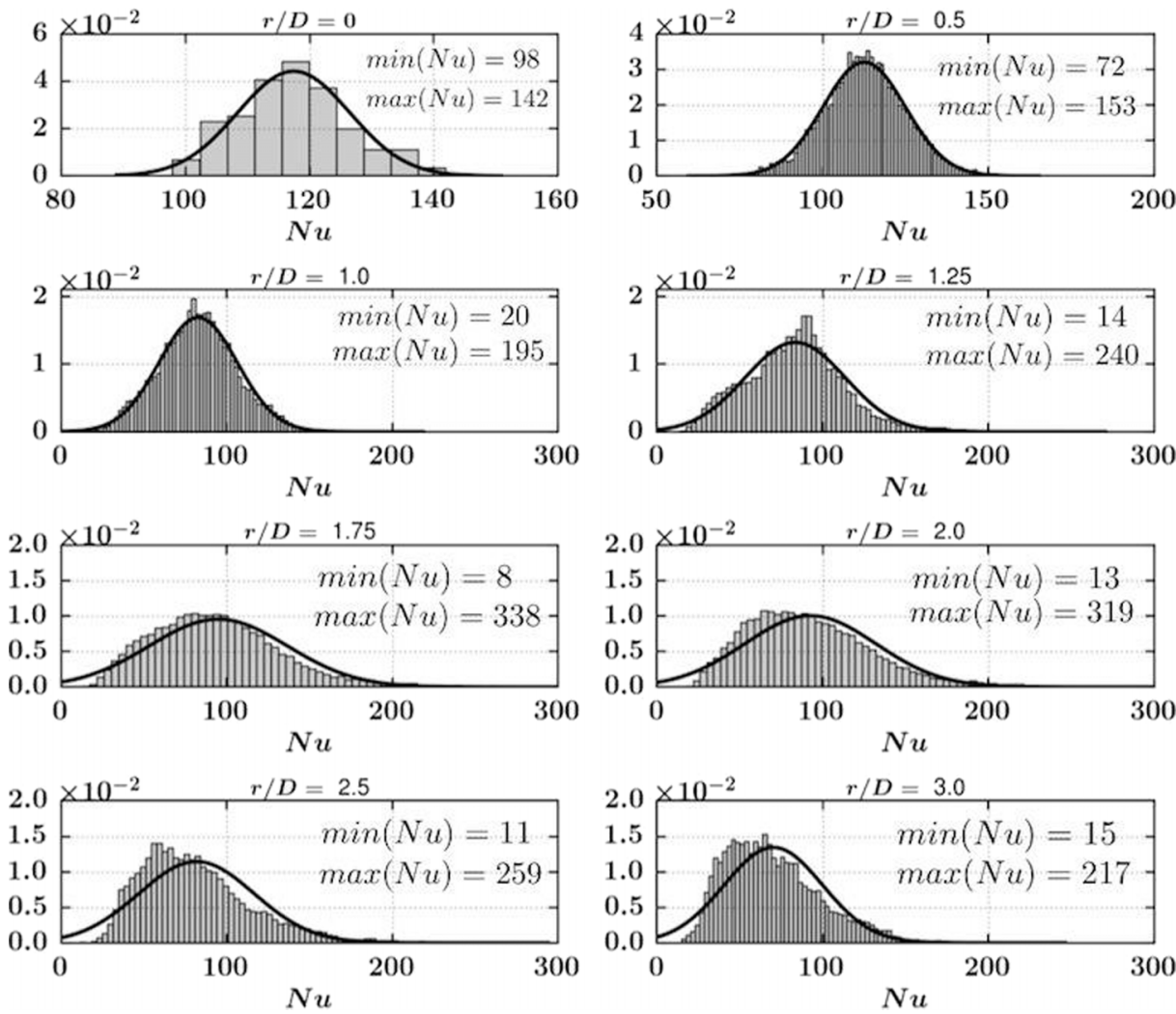

FIG. 23. PDF of the Nusselt number values, $P(N u)$, on the plate at 8 locations along the radius. (-): Gaussian PDF with the same mean and RMS values as the Nusselt data set.

confirms that the generation of the secondary peak is due to the strong intermittent thermal events, i.e., the asymmetric part of the PDFs. One can also note that after $r / D=2$, the two curves are not superimposed. This reflects the contribution to the mean value of the persistent and relatively strong thermal events even after the second peak.

Finally, these results have to be linked with the flow dynamics to propose a scenario explaining the heat transfer enhancement. In agreement with the documented experimental results, the primary vortices impinge the wall as expected for this low nozzle to plate separation, ${ }^{7}$ inducing an adverse pressure gradient and the separation of the boundary layer ${ }^{7,14}$ identified by the emergence of region $\mathrm{B}$ on the skewness of the temporal distribution of the pressure presented in Fig. 12. The separation

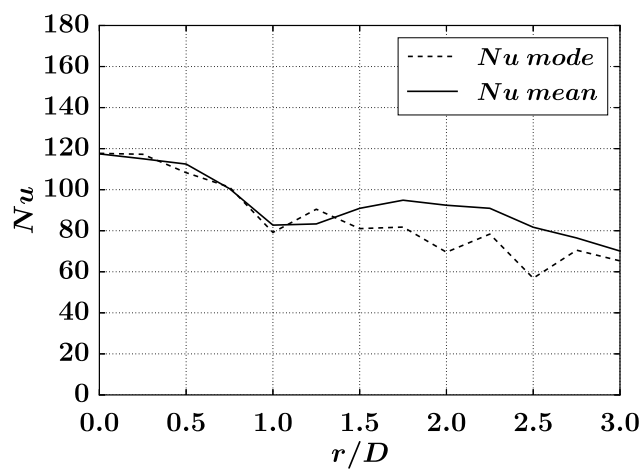

FIG. 24. Nusselt number $N u$ as a function of the radial distance from the jet axis $r / D$. (-): Temporally and azimuthally averaged $N u,(--)$ : mode value of the $N u$ data set. 
process induces low heat transfer events as shown by the Nusselt PDFs and causes the local minimum in the mean heat transfer distribution where the separation evolves into a secondary vortex. These results are coherent with previous findings ${ }^{22,31,32}$ that have shown a coincidence between the instantaneous position of the Nusselt minimum and the instantaneous location where distinct secondary vortices appear. Secondary vortices, in addition to the primary vortices, organize the mixing near the wall producing preferentially a cold fluid flux towards the plate in the region of heat transfer enhancement. After the local minimum, the most probable event is the cold fluid injection generating high heat transfer events confirming the results of Dairay et al. ${ }^{21}$ It seems also that the secondary vortices compensate the low heat transfer events induced by the separation and produce the strongest thermal events, i.e., cold front B which combined with the intermittent unperturbed wall jet, i.e., cold front A, leads to the mean heat transfer enhancement. Near the location of the secondary peak, the rebound of the primary vortices is highlighted in agreement with recent experimental aerothermal investigation, ${ }^{34}$ while the secondary vortices seem to stay close to the wall. At the location where the primary and secondary vortices could not be distinguished from one another on the skewness map (see Fig. 12), the cold fluid injection and the hot fluid ejection converge to the same probability and the mean heat transfer enhancement stops, although the Nusselt PDFs remain skewed beyond the secondary peak up to $r / D=3$. The positive skewness is linked to residual strong thermal events intermittent in time and space. Indeed as seen in Fig. 19, circular patterns with a reduced azimuthal coherence are present at $r / D \approx 1.9$ for the two instantaneous snapshots. These residual strong thermal events are also related to the secondary vortex that is subject to a potential interaction with the rebounding primary vortex which reduces circumferential coherence and leads to the transition of the flow field to a fully turbulent state in the same way as for the impingement of a single vortex ring on a flat plate. ${ }^{12,64,65}$

\section{CONCLUSIONS}

A wall-resolved LES of a single unconfined round jet normally impinging on a flat plate has been performed to investigate the link between the Nusselt secondary peak and the near-wall flow dynamics. The results have been validated against experimental data available in the literature. Prior to detailed flow analyses, the numerical uncertainties issued by the SGS model and the grid resolution are reported for the mean and RMS velocity profiles, the wall shear stress, and the wall heat transfer. Based on this validated numerical database, the flow dynamics and the heat transfer process are investigated to establish a link between the vortical structures present in impinging jet flows and the non-monotonic mean Nusselt distribution. For this configuration, primary and secondary vortices are evidenced using iso-surfaces of Q-criterion in agreement with the previous studies. ${ }^{21}$ The skewness of the temporal distribution of the pressure allowed to highlight the rebound of the primary vortices that is observed for a single vortex ring impinging on a flat plate but that is more difficult to characterize in a fully turbulent impinging jet flow. It appears that this rebound is the event that stops the mean heat transfer enhancement as the secondary peak in the mean Nusselt distribution is located at the same position. In addition, the skewness of the pressure also allowed to identify the location of the initiation of the unsteady separation which coincides with the location of the local minimum. This point analyzed jointly with the Nusselt PDFs brings a clear evidence that this separation generates low heat transfer events, due to fluid pockets that are heated by the plate, which contribute to the local minimum of the mean Nusselt radial distribution. This observation goes in the direction of the scenario proposed by Hadžiabdić and Hanjalić ${ }^{22}$ to explain the dip between the two peaks. A correlation is also observed between the location of the local minimum and the intermittent generation of a strong cold front related to a cold fluid injection induced by the secondary vortices. These results are coherent with Dairay et al. ${ }^{21}$ which have highlighted a cold fluid flux towards the wall in the vicinity of a cold spot using conditional averaging. From the Nusselt PDFs, it is concluded that the heat transfer enhancement is the consequence of an imbalance between high and low Nusselt values caused by the secondary structures, which appears to be the main mechanism inducing the positive skewness, confirming once again the scenario proposed by Hadžiabdić and Hanjalić. ${ }^{22}$ Finally, the rebound of the primary vortices is found to be the event that causes the second peak representing the end of the heat transfer enhancement. 


\section{ACKNOWLEDGMENTS}

The authors acknowledge CINES of GENCI for giving access to HPC resources under the allocation 2016-x20162a6074. The authors are grateful to Safran Helicopter Engines for funding this work.

${ }^{1}$ J.-C. Han and L. M. Wright, "Enhanced internal cooling of turbine blades and vanes," in The Gas Turbine Handbook (U.S. National Energy Technology Laboratory, Morgantown, WV, 2007), pp. 321-352.

${ }^{2}$ K. Jambunathan, E. Lai, M. A. Moss, and B. L. Button, "A review of heat transfer data for single circular jet impingement," Int. J. Heat Fluid Flow 13, 106-115 (1992).

${ }^{3}$ R. Viskanta, "Heat transfer to impinging isothermal gas and flame jets," Exp. Therm. Fluid Sci. 6, 111-134 (1993).

${ }^{4}$ A. Dewan, R. Dutta, and B. Srinivasan, "Recent trends in computation of turbulent jet impingement heat transfer," Heat Transfer Eng. 33, 447-460 (2012).

${ }^{5}$ H. Martin, "Heat and mass transfer between impinging gas jets and solid surfaces," in Advances in Heat Transfer (Elsevier Ltd., New york, 1977), Vol. 13, pp. 1-60.

${ }^{6}$ S. C. Crow and F. H. Champagne, "Orderly structure in jet turbulence," J. Fluid Mech. 48, 547-591 (1970).

${ }^{7}$ C. Popiel and O. Trass, "Visualization of a free and impinging round jet," Exp. Therm. Fluid Sci. 4, 253-264 (1991).

${ }^{8}$ C. Cornaro, A. S. Fleischer, and R. J. Goldstein, "Flow visualization of a round jet impinging on cylindrical surfaces," Exp. Therm. Fluid Sci. 20, 66-78 (1999).

${ }^{9}$ A. Yule, "Large-scale structure in the mixing layer of a round jet," J. Fluid Mech. 89, 413 (1978).

${ }^{10}$ A. K. M. F. Hussain and K. B. M. Q. Zaman, "The 'preferred mode' of the axisymmetric jet," J. Fluid Mech. 110, 39-71 (1981).

${ }^{11}$ C. Chu, C. Wang, and C. Hsieh, “An experimental investigation of vortex motions near surfaces,” Phys. Fluids A 5, 662-676 (1993).

12 J. D. A. Walker, C. R. Smith, A. W. Cerra, and T. L. Doligalski, “The impact of a vortex ring on a wall,” J. Fluid Mech. 41, 99-140 (1987).

${ }^{13}$ N. Uddin, S. Neumann, and B. Weigand, "LES simulations of an impinging jet: On the origin of the second peak in the Nusselt number distribution,” Int. J. Heat Mass Transfer 57, 356-368 (2013).

${ }^{14}$ N. Didden and C.-M. Ho, "Unsteady separation in a boundary layer produced by an impinging jet," J. Fluid Mech. 160, 235 (1985).

${ }^{15}$ M. Angioletti, R. Di Tommaso, E. Nino, and G. Ruocco, "Simultaneous visualization of flow field and evaluation of local heat transfer by transitional impinging jets," Int. J. Heat Mass Transfer 46, 1703-1713 (2003).

${ }^{16} \mathrm{~J}$. W. Baughn and S. Shimizu, "Heat transfer measurements from a surface with uniform heat flux and an impinging jet," J. Heat Transfer 111, 1096-1098 (1989).

${ }^{17}$ R. Gardon and J. Akfirat, "The role of turbulence in determining the heat-transfer characteristics of impinging jets," Int. J. Heat Mass Transfer 8, 1261-1272 (1965)

${ }^{18}$ K. Kataoka, R. Sahara, H. Ase, and T. Harada, "Role of large scale coherent structures in impinging jet heat transfer," J. Chem. Eng. Jpn. 20, 71-76 (1987).

${ }^{19}$ T. O’Donovan and D. Murray, "Jet impingement heat transfer Part I: Mean and root-mean-square heat transfer and velocity distributions," Int. J. Heat Mass Transfer 50, 3291-3301 (2007).

${ }^{20}$ S. Roux, M. Fenot, G. Lalizel, L. E. Brizzi, and E. Dorignac, "Evidence of flow vortex signatures on wall fluctuating temperature using unsteady infrared thermography for an acoustically forced impinging jet," Int. J. Heat Fluid Flow 50, 38-50 (2014).

${ }^{21}$ T. Dairay, V. Fortuné, E. Lamballais, and L.-E. Brizzi, "Direct numerical simulation of a turbulent jet impinging on a heated wall," J. Fluid Mech. 764, 362-394 (2015).

${ }^{22}$ M. Hadžiabdić and K. Hanjalić, "Vortical structures and heat transfer in a round impinging jet," J. Fluid Mech. 596, 221-260 (2008).

${ }^{23}$ H. Hattori and Y. Nagano, "Direct numerical simulation of turbulent heat transfer in plane impinging jet," Int. J. Heat Fluid Flow 25, 749-758 (2004).

${ }^{24}$ F. Shum-kivan, F. Duchaine, and L. Gicquel, "Large-eddy simulation and conjugate heat transfer in a round impinging jet," in ASME Turbo Expo 2014: Turbine Technical Conference and Exposition (ASME, 2014).

25 J. Lee and S.-J. Lee, "Stagnation region heat transfer of a turbulent axisymmetric jet impingement," Exp. Heat Transfer 12, 137-156 (1999).

${ }^{26}$ N. T. Obot, A. S. Majumdar, and W. J. M. Douglas, The Effect of Nozzle Geometry on Impingement Heat Transfer Under a Round Turbulent Jet (American Society of Mechanical Engineers, 1979).

${ }^{27}$ S. Roux, M. Fénot, G. Lalizel, L.-E. Brizzi, and E. Dorignac, "Experimental investigation of the flow and heat transfer of an impinging jet under acoustic excitation," Int. J. Heat Mass Transfer 54, 3277-3290 (2011).

${ }^{28}$ D. W. Colucci and R. Viskanta, "Effect of nozzle geometry on local convective heat transfer to a confined impinging air jet," Exp. Therm. Fluid Sci. 13, 71-80 (1996).

${ }^{29}$ L. F. G. Geers, K. Hanjalic, and M. J. Tummers, "Wall imprint of turbulent structures and heat transfer in multiple impinging jet arrays," J. Fluid Mech. 546, 255-284 (2006).

${ }^{30}$ D. Lytle and B. Webb, "Air jet impingement heat transfer at low nozzle-plate spacings," Int. J. Heat Mass Transfer 37, 1687-1697 (1994).

${ }^{31}$ Y. M. Chung, K. H. Luo, and N. D. Sandham, "Numerical study of momentum and heat transfer in unsteady impinging jets," Int. J. Heat Fluid Flow 23, 592-600 (2002).

${ }^{32}$ W. Rohlfs, H. Haustein, O. Garbrecht, and R. Kneer, "Insights into the local heat transfer of a submerged impinging jet Influence of local flow acceleration and vortex-wall interaction,” Int. J. Heat Mass Transfer 55, 7728-7736 (2012). 
${ }^{33}$ M. Fenot, J. J. Vullierme, and E. Dorignac, "Local heat transfer due to several configurations of circular air jets impinging on a flat plate with and without semi-confinement," Int. J. Therm. Sci. 44, 665-675 (2005).

${ }^{34}$ P. Grenson, O. Léon, P. Reulet, and B. Aupoix, "Investigation of an impinging heated jet for a small nozzle-to-plate distance and high Reynolds number: An extensive experimental approach,” Int. J. Heat Mass Transfer 102, 801-815 (2016).

${ }^{35}$ M. J. Tummers, J. Jacobse, and S. G. J. Voorbrood, "Turbulent flow in the near field of a round impinging jet," Int. J. Heat Mass Transfer 54, 4939-4948 (2011).

${ }^{36}$ T. Schonfeld and M. Rudgyard, "Steady and unsteady flows simulations using the hybrid flow solver AVBP," AIAA J. 37, 1378-1385 (1999).

${ }^{37}$ O. Colin and M. Rudgyard, “Development of high-order Taylor-Galerkin schemes for LES,” J. Comput. Phys. 162, 338-371 (2000).

38 J. Donea and A. Huerta, Finite Element Methods for Flow Problems (John Wiley \& Sons, Ltd., 2003).

${ }^{39}$ F. Duchaine, N. Maheu, V. Moureau, G. Balarac, and S. Moreau, "Large-eddy simulation and conjugate heat transfer around a low-Mach turbine blade," J. Turbomach. 136, 051015 (2013).

${ }^{40} \mathrm{C}$. Koupper, "Unsteady multi-component simulations dedicated to the impact of the combustion chamber on the turbine of aeronautical gas turbines," Ph.D. thesis, INP Toulouse, 2015.

${ }^{41}$ R. Fransen, "LES based aerothermal modeling of turbine blade cooling systems," Ph.D. thesis, INP Toulouse, 2013.

42 D. Papadogiannis, "Coupled large eddy simulations of combustion - turbine interactions," Ph.D. thesis, INP Toulouse, 2015.

${ }^{43}$ L. Y. M. Gicquel, G. Staffelbach, and T. Poinsot, "Large eddy simulations of gaseous flames in gas turbine combustion chambers," Prog. Energy Combust. Sci. 38, 782-817 (2012).

${ }^{44}$ S. Mendez and F. Nicoud, "Large-eddy simulation of a bi-periodic turbulent flow with effusion," J. Fluid Mech. 598, 27-65 (2008).

${ }^{45}$ S. B. Pope, Turbulent Flows (Cambridge University Press, New York, 2000).

${ }^{46}$ F. Nicoud and F. Ducros, "Subgrid-scale stress modelling based on the square of the velocity gradient tensor," Flow, Turbul. Combust. 62, 183-200 (1999).

${ }^{47}$ J. Smagorinsky, "General circulation experiments with the primitive equations. I. The basics experiment," Mon. Weather Rev. 91, 99-164 (1963).

${ }^{48}$ T. J. Poinsot and S. K. Lele, "Boundary conditions for direct simulations of compressible viscous flows," J. Comput. Phys. 101, 104-129 (1992).

${ }^{49}$ G. Lodato, L. Vervisch, and P. Domingo, "A compressible wall-adapting similarity mixed model for large-eddy simulation of the impinging round jet," Phys. Fluids 21, 035102 (2009).

${ }^{50}$ D. Cooper, D. Jackson, B. Launder, and G. Liao, "Impinging jet studies for turbulence model assessment. I. Flow field experiments,” Int. J. Heat Mass Transfer 36, 2675-2684 (1993).

${ }^{51}$ N. Guézennec and T. Poinsot, "Acoustically nonreflecting and reflecting boundary conditions for vortcity injection in compressible solvers," AIAA J. 47, 1709-1722 (2009).

${ }^{52}$ V. Granet, O. Vermorel, T. Léonard, L. Gicquel, and T. Poinsot, "Comparison of nonreflecting outlet boundary conditions for compressible solvers on unstructured grids," AIAA J. 48, 2348-2364 (2010).

${ }^{53}$ P. Sagaut, Large Eddy Simulation for Incompressible Flows (Springer-Verlag, 2000).

${ }^{54}$ M. Bovo and L. Davidson, "Direct comparison of les and experiment of a single-pulse impinging jet," Int. J. Heat Mass Transfer 88, 102-110 (2015)

${ }^{55}$ F. Nicoud, H. B. Toda, O. Cabrit, S. Bose, and J. Lee, "Using singular values to build a subgrid-scale model for large eddy simulations," Phys. Fluids 23, 085106 (2011).

${ }^{56}$ C. Koupper, L. Gicquel, F. Duchaine, and G. Bonneau, "Advanced combustor exit plane temperature diagnostics based on large eddy simulations: Going further than the radial temperature distribution factor," Flow, Turbul. Combust. 95, 79-96 (2015).

${ }^{57}$ K. P. Balanda and H. L. MacGillivray, "Kurtosis: A critical review," Am. Stat. 42, 111-119 (1988).

${ }^{58}$ L. T. DeCarlo, "On the meaning and use of kurtosis," Psychol. Methods 2, 292-307 (1997).

${ }^{59}$ K. D. Hopkins and D. L. Weeks, "Tests for normality and measures of skewness and kurtosis: Their place in research reporting," Educ. Psychol. Meas. 50, 717-729 (1990).

${ }^{60}$ H. Tennekes and J. L. Lumley, A First Course in Turbulence (MIT Press, 1972).

${ }^{61}$ C.-M. Ho and N. S. Nosseir, "Dynamics of an impinging jet. I. The feedback phenomenon,” J. Fluid Mech. 105, 119 (1981).

${ }^{62}$ K. B. M. Q. Zaman and a. K. M. F. Hussain, "Taylor hypothesis and large-scale coherent structures," J. Fluid Mech. 112, 379-396 (1981).

${ }^{63}$ D. J. C. Dennis and T. B. Nickels, "On the limitations of Taylor's hypothesis in constructing long structures in a turbulent boundary layer," J. Fluid Mech. 614, 197 (2008).

${ }^{64}$ A. Cerra and C. R. Smith, Experimental observation of vortex ring interaction with the fluid adjacent to a surface, Technical Report, Lehigh University, 1983.

${ }^{65}$ P. Orlandi and R. Verzicco, "Vortex rings impinging on walls: Axisymmetric and three-dimensional simulations," J. Fluid Mech. 256, 615-646 (1993). 